Cattaleeya Pattamaprom

Ronald G. Larson

Timothy J. Van Dyke

\section{Quantitative predictions of linear viscoelastic rheological properties of entangled polymers}

Received: 23 December 1999

Accepted: 28 March 2000

\begin{abstract}
The "dual constraint" model developed by Mead, Van Dyke et al. is here extended by inclusion of "early-time" contourlength fluctuations and constraintrelease Rouse relaxation, and then evaluated by comparing its predictions with literature data for over 50 different linear and star polymers. By combining the reptation model of Doi and Edwards with contourlength fluctuations and constraint release, the model provides a systematic approach to prediction of the rheological properties of polymers.
\end{abstract}

\begin{abstract}
The parameters are taken from the literature and used consistently for linear polymers, star polymers, and their mixtures having the same chemical compositions. In most cases, the predictions of the model appears to agree well with data for monodisperse, bidisperse, and polydisperse linear and star polymers, except at low molecular weights.
\end{abstract}

Key words Linear viscoelastic . Double reptation - Constraint release $\cdot$ Linear polymers $\cdot$ Star polymers

\section{Introduction}

The first detailed molecular model for predicting rheological properties of well entangled polymers was originated by de Gennes (1971) and developed into a full constitutive theory by Doi and Edwards (1978, 1979). This model is based on the notion of reptation in a conceptual "tube" of constraints imposed by the mesh of surrounding polymer molecules. While the DoiEdwards theory successfully predicts the nonlinear rheological response of entangled polymers in step strains, it has several drawbacks in both linear and nonlinear regimes. Since an understanding of the linear regime is foundational for that of the nonlinear regime, the accuracy of predictions in the linear regime is crucial. The drawbacks of the Doi-Edwards prediction in the linear regime are that:

1. It predicts the low-shear-rate Newtonian viscosity to scale as $\mathbf{M}^{3}$ instead of $\mathbf{M}^{3.4}$ which is observed experimentally, where $\mathrm{M}$ is the polymer molecular weight.
2. For frequencies beyond the crossover point of $\mathrm{G}^{\prime}$ and $G^{\prime \prime}$, the Doi-Edwards theory predicts that the loss modulus $\left(\mathrm{G}^{\prime \prime}\right)$ decreases in proportion to $\omega^{-0.5}$ which is steeper than the observed behavior, for which the exponent ranges between 0 and -0.25 depending on the molecular weight of the polymer.

3. The Doi-Edwards theory fails to predict a highfrequency upturn in $G^{\prime}$ and $G^{\prime \prime}$.

These discrepancies in the Doi-Edwards predictions are probably due to nonreptative mechanisms, mainly contour-length fluctuations and Rouse relaxations.

Milner and McLeish (1997, 1998) have proposed improved models for predicting the linear rheological properties of monodisperse linear and star polymers. Their theory for monodisperse linear polymers combines reptation, contour-length fluctuations, and Rouse relaxation processes. (Milner and McLeish 1997) Their models for monodisperse and bidisperse star polymers combine contour-length fluctuations with a tube-renewal process called "dynamic dilution." They have also recently proposed a theory for mixtures of monodisperse 
linear with monodisperse star polymers. (Milner et al. 1998) While these models seem successfully to describe monodisperse linear, monodisperse, and bidisperse star data, and mixtures of monodisperse linear and star polymers, there is a need for a single "universal" framework capable of encompassing polydisperse linear and star molecules, or mixtures of linear and star molecules. Developing such a theory is challenging because the theory for star polymers describes constraint release entirely in terms of a "tube dilution" process, which is an inappropriate description of constraint release for linear polymers.

Recently, Mead, Van Dyke et al. (2000) have developed the "dual constraint model," in which an approximate treatment of constraint release processes combines both "double reptation" and "tube dilution" concepts. While the "tube dilution" ideas in this model are not fully self consistent, the model does have the virtue that it is, in principle, equally applicable to linear and star polymers, whether monodisperse or polydisperse, and to mixtures of linears and stars. In principle, it is also extendible to the nonlinear regime.

In addition, the "dual constraint" model involves no parameters beyond the friction coefficient $\zeta$ of Rouse theory and the tube diameter $a$ (or, equivalently, the plateau modulus $\mathrm{G}_{\mathrm{N}}^{0}$ ) of reptation theory. Thus, once these two parameters have been specified (from the literature, for example), predictions for well entangled polymers can be made for linear or star polymers of arbitrary molecular weight, or for polydisperse polymers, without needing to specify other parameters.

In this paper, we extend the "dual constraint" model by incorporating (a) early-time contour-length fluctuations (Milner and McLeish 1997, 1998) and (b) "constraint-release Rouse" processes (Viovy et al. 1991); we then thoroughly test the extended dual constraint model in the linear viscoelastic regime by using it to predict sets of rheological data from different labs, including virtually all sets of linear viscoelastic data from the literature for well characterized monodisperse and bidisperse linear and star polymers. Where available, we use literature values for the parameters, $\zeta, a$ and $\mathrm{G}_{\mathrm{N}}^{0}$. The behaviors of linear and star polymers of the same chemical composition are then correlated by using the same model parameters for both architectures. In addition, we also test the model by comparing its predictions to linear viscoelastic data for well defined polydisperse samples produced by mixing together a large number of well defined monodisperse fractions. By confronting the model with many different data sets in the literature, and by using the same input parameters to predict data from a wide variety of sources, we are able to evaluate thoroughly and objectively the validity of the model, and to provide a standard of comparison against which any future improved model can be judged.

\section{Theoretical background}

The "dual constraint" model that we will test is that of Mead, Van Dyke, et al., who incorporated two forms of constraint release into the dynamics of entangled polymers. The first form of constraint release is "double diffusion" which augments the "double reptation" theory of constraint release by inclusion of fluctuation as a mechanism of constraint release. This first mode of constraint release has been found successfully to account for the effects of polydispersity on the relaxation of polydisperse linear chains in most cases.

The second form of constraint release is "dynamic dilution," which was introduced by Ball and McLeish (1989) to describe the self-consistent time-dependent loosening of the effective entanglement network, or widening of the tube, that is realized when the relaxation of monodisperse star polymers is considered on ever longer time-scales. This second form of constraint release is required for stars, because even a monodisperse star has such a wide range of relaxation times that over the time-scale required for the last interior part of an arm to relax, the tip of that arm has relaxed so many times that it acts more like a solvent than a real obstacle to motion of other chains.

Both forms of constraint release assume that the constraint releases lead to immediate relaxation of the constrained chain (for double diffusion) or immediate expansion of the chain to match with the wider tube (for dynamic dilution). This assumption has been found to be acceptable for most cases except when dilute chains with long relaxation times (i.e., long chains) are in the matrix of chains with much shorter relaxation time (i.e., short chains) (Milner 1996). The failure is expected because the dilute long chains cannot explore the dilated tube as fast as the dilation process occurs by relaxation of short chains in the matrix. This case (dilute long chains in a matrix of short chains) has been studied by Watanabe et al. (1985, 1991) and Montfort et al. (1984), and theoretically by Viovy et al. (1991). The result can be described by considering so-called "constraint-release Rouse" motions, which are Rouse-like motions of the long chain in a "supertube" produced by relaxation of the short chains.

In order to make the model applicable in all cases, the extended dual constraint model described here combines both forms of constraint release together with the constraint-release Rouse process into a single formalism. It does so by solving twice a diffusion equation for reptation and/or fluctuation of linear or star polymers. The equation is first solved in a fixed tube, i.e., in the absence of constraint release. This first solution to the diffusion equation supplies a single quantity $\psi^{*}$, the time dependent fraction of the entanglements that would survive over a period of time $t$ if each chain relaxed in a fixed matrix. This function is then corrected with the constraint-release Rouse process when $\psi^{*}(t)$ relaxes faster than Rouse motion of the chain, and the corrected function named $\phi^{*}(t)$. $\phi^{*}(t)$ is then used as a time-dependent dilution factor which affects fluctuations in a second solution of the diffusion equation. After solving the diffusion equation this second time, with dynamic dilution included via $\phi^{*}(t)$, the resulting survival probability of the chain is then obtained. The survival probability of the constraint surrounding the chain is the same as that of the chain except that the former is corrected for the constraint-release Rouse process. The survival probability of the chain is multiplied by that of the constraint to account for double diffusion and then multiplied by the plateau modulus to get the relaxation modulus. Finally, the high-frequency Rouse relaxation modes are added.

In what follows, we lay out the equations solved here; further discussion of these equations, and justification for them, are provided by Mead, Van Dyke et al. (2000).

\section{A. Reptation and fluctuation in a fixed matrix}

The linear viscoelastic constitutive equation for highly entangled polymers proposed here is based on the tube model of Doi and 
Edwards (1978, 1979, 1986). For oscillatory deformation at low and moderate frequencies, the major relaxation mechanisms for linear polymers are reptation, contour-length fluctuation, and constraint release. For star polymers, the arms are joined together, preventing reptation. Thus, contour-length fluctuation and constraint release are the only relaxation mechanisms for stars at low and moderate frequencies. At high frequencies, Rouse processes are important for both architectures.

Reptation can be thought of as the diffusion of a chain out of a tube; therefore, it can be represented by a diffusion equation for the tube-survival probability. Contour-length fluctuation is a relaxation process induced by thermal fluctuation of the tube length with time. Including both reptation and contour-length fluctuation, the diffusion equation for linear polymers subjected to a small step strain at time $t=0$ is (Mead, Van Dyke et al. 2000).

$\frac{\partial p_{i}^{*}\left(s_{i}, t\right)}{\partial t}=\frac{D_{i}}{L_{i}^{2}} \frac{\partial^{2} p_{i}^{*}\left(s_{i}, t\right)}{\partial s_{i}^{2}}-\frac{p_{i}^{*}\left(s_{i}, t\right)}{\tau_{\xi, i}^{*}\left(s_{i}\right)}$

The initial and bounary conditions are $p_{i}^{*}=1$ at $t=0$, and $p_{i}^{*}=0$, at the chain end. The first term on the right of Eq. (1) represents the reptation mechanism and the second term is for contour-length fluctuation. The equation is the same for star polymers except for star, the reptation term is removed. The subscript $i$ denotes a linear chain of molecular weight $\mathbf{M}_{i}$ or the arm molecular weight for a star polymer. In Eq. (1), $p_{i}^{*}\left(\mathrm{~s}_{i}, t\right)$ is the survival probability of a tube segment occupied by a chain of type $i$ as a function of time $t$ and contour distance $s_{\mathrm{i}}$, where $s_{\mathrm{i}}$ ranges from 0 at the center of a linear polymer chain or the branch point of a star to $1 \frac{1 / 2}{2}$ at the chain end of a linear, or to 1 at the chain end of a star. $D_{i}$, the curvilinear diffusion coefficient, is defined as $L_{i}^{2} / \tau_{\mathrm{d}, i} \pi^{2}$, where $L_{i}$ is the average contour length of the tube and $\tau_{\mathrm{d}, \mathrm{i}}$ is the reptation time constant (Doi and Edwards 1986). $L_{i}$ equals $\mathrm{N}_{i} \mathrm{~b}^{2} / a$, where $a$ is the tube diameter, $\mathrm{b}$ is the effective polymer statistical segment length, and $\mathrm{N}_{i}$ is the number of monomers composing a chain of type $i$. The relationship between $a$ and $\mathrm{b}$ is given by $\mathrm{b}=a\left(\sqrt{\mathrm{M}_{\mathrm{o}}} / \mathrm{M}_{\mathrm{e}}\right)$, and $\mathrm{N}_{i}$ can be rewritten as $\mathbf{M}_{i} / \mathbf{M}_{\mathrm{o}}$, where $\mathbf{M}_{\mathrm{o}}$ is the monomer molecular weight and $\mathbf{M}_{\mathrm{e}}$ is the molecular weight between two entanglements. $\tau_{\mathrm{d}, i}$ is given by $\tau_{\mathrm{d}, i}=\left(\zeta \mathrm{N}_{i}^{3} \mathrm{~b}^{4}\right) /\left(\pi^{2} \mathrm{k}_{\mathrm{b}} \mathrm{T} a^{2}\right)$ where $\mathrm{k}_{\mathrm{b}}$ is the Boltzmann constant and $\mathrm{T}$ is the absolute temperature (Doi and Edwards 1986, p 196).

The second term represents contour-length fluctuations. The relaxation time for contour-length fluctuations, $\tau_{\xi, i}^{*}$, accounts for the activated relaxation described by Doi and Kuzuu (1980), Pearson and Helfand (1984), Doi and Edwards (1986), and Mead, Van Dyke et al. (2000). This activated relaxation, which has for its prefactor the longest Rouse relaxation time, is accurate for deep fluctuations. Shallow fluctuations, however, only drag a small bit of the chain, and hence are much faster than predicted by the formula for activated relaxation. A formula for these shallow, fast, fluctuation modes has been derived from Milner and McLeish (1997, 1998). Borrowing from this work, we have two expressions, one for shallow $\left(\tau_{\text {early,i }}\right)$ and one for deep $\left(\tau_{\text {late }, i}^{*}\right)$ fluctuations:

$$
\begin{aligned}
\tau_{\text {early }, i}\left(\mathrm{~s}_{i}\right)= & \frac{225}{256} \pi^{3} \frac{\tau_{\mathrm{R}, i}}{\mathrm{c}^{2}}\left(1-\mathrm{cs}_{i}\right)^{4}\left(\frac{\mathrm{N}_{\mathrm{en}, i}}{\mathrm{c}}\right)^{2} \\
& \text { and } \tau_{\text {late }, i}^{*}\left(\mathrm{~s}_{i}\right)=\frac{\tau_{\mathrm{R}, i}}{\mathrm{c}^{2}} \exp \left(\mathrm{U}_{\left(\mathrm{s}_{i}\right)}^{*}\right)
\end{aligned}
$$

Here $\mathrm{c}$ is a prefactor which equals 1 for star polymers and 2 for linear polymers (since a linear polymer has two fluctuating chain ends). $\mathrm{N}_{\mathrm{en}, i} \equiv \mathrm{M}_{i} / \mathrm{M}_{\mathrm{e}}$ is the number of entanglements per chain for linear polymers or per arm for stars. $\tau_{\mathrm{R}, i}$ is the longest Rouse relaxation time of a chain defined by $\tau_{\mathrm{R}, i}=\zeta \mathrm{N}_{i}^{2} \mathrm{~b}_{i}^{2} / 6 \pi^{2} \mathrm{k}_{\mathrm{b}} \mathrm{T}$, which is smaller than the rotational relaxation time $\tau_{\mathrm{r}}$ from Doi and Edwards by a factor of two (Doi and Edwards 1986, p 115). Thus, $\tau_{\mathrm{R}, i}$ is related to $\tau_{\mathrm{d}, i}$ by $\tau_{\mathrm{R}, i}=\tau_{\mathrm{d}, i} /\left(6 \mathrm{~N}_{\mathrm{en}, i}\right)$.

$\mathrm{U}^{*}\left(\mathrm{~s}_{i}\right)$ is the activation energy for chain retraction given by

$\mathrm{U}^{*}\left(\mathrm{~s}_{i}\right)=\frac{15}{8} \frac{\mathrm{N}_{\mathrm{en}, i}}{\mathrm{c}}\left(1-\mathrm{cs}_{i}\right)^{2}$
The quick fluctuation mode of chain ends ( $s_{\mathrm{i}}$ close to 1$)$ is controlled by $\tau_{\text {early }, i}$ and gradually changes to the normal activation mode $\tau_{\mathrm{late}, i}^{*}$ toward the center ( $s_{i}$ close to 0 ). Therefore, $\tau_{\xi, i}^{*}$ in Eq. (1) equals $\tau_{\text {early }, i}$ for large $s_{i}$ and $\tau_{\text {late }, i}^{*}$ for small $s_{i}$. We propose here a simple crossover function that splices these two functions together with a transition zone over which a geometric average of the two is used. The crossover function from $\tau_{\text {early }, i}$ to $\tau_{\text {late }, i}^{*}$ along the chain follows the solid line in Fig. 1, which is given by

$$
\begin{aligned}
\tau_{\xi, i}^{*} & =\tau_{\text {early }, i} & & \text { when }\left(1-s_{i}\right)<\mathrm{C}_{1}^{*} \\
\tau_{\xi, i}^{*} & =\tau_{\text {early }, i} \cdot \tau_{\text {late }, i}^{*} & & \text { when } \mathrm{C}_{1}^{*}<\left(1-s_{i}\right)<\mathrm{C}_{2}^{*} \\
\tau_{\xi, i}^{*} & =\tau_{\text {late }, i}^{*} & & \text { when }\left(1-s_{i}\right)<\mathrm{C}_{2}^{*}
\end{aligned}
$$

where $\mathrm{C}_{1}^{*}$ is the first crossover position of $\tau_{\mathrm{early}, i}$ and $\tau_{\mathrm{late}, i}^{*}$ close to the chain end, and $\mathrm{C}_{2}^{*}$ is the second crossover point deeper inside the tube. Close to the chain end where $\left(1-s_{i}\right)<\mathrm{C}_{1}^{*}, \tau_{\xi i}^{*}$ takes the value of $\tau_{\text {early,i }}$ and deep inside the tube where $\left(1-s_{i}\right)>\mathrm{C}_{2}^{*}, \tau_{\xi, i}^{*}$ is controlled by $\tau_{\text {late }, i}^{*}$ For the tube segments between $\mathrm{C}_{1}^{*}$ and $\mathrm{C}_{2}^{*}, \tau_{\xi, i}^{*}$ is taken to be the geometric average of the two functions of $s_{\mathrm{i}}$.

The overall survival probability $\psi_{i}^{*}(t)$ of a tube occupied by chain $i$ is then calculated from $p_{i}^{*}\left(s_{\mathrm{i}}, t\right)$ by

$\psi_{i}^{*}(t)=\int_{0}^{1} p_{i}^{*}\left(s_{i}, t\right) \mathrm{ds}_{i}$

and the average tube survival probability $\psi_{i}^{*}(t)$ of all chains is

$\psi^{*}(t)=\Sigma w_{i} \psi_{i}^{*}(t)$

where $w_{i}$ is the weight fraction of the chains of length $\mathrm{L}_{i}$. We obtain $\psi^{*}(t)$ numerically by solving for $p_{i}^{*}\left(s_{i}, t\right)$ using a Crank-Nicolson method, and then converting to $\psi^{*}(t)$ and $\psi^{*}(t)$ by means of Eqs. (5) and (6), respectively. Note that $\psi^{*}(t)$ is calculated when constraint release is not present.

The approximate constraint-release Rouse process is then activated when $\psi^{*}(t)$ decreases faster than a Rouse relaxation process would permit. A detailed explanation of the constraint-

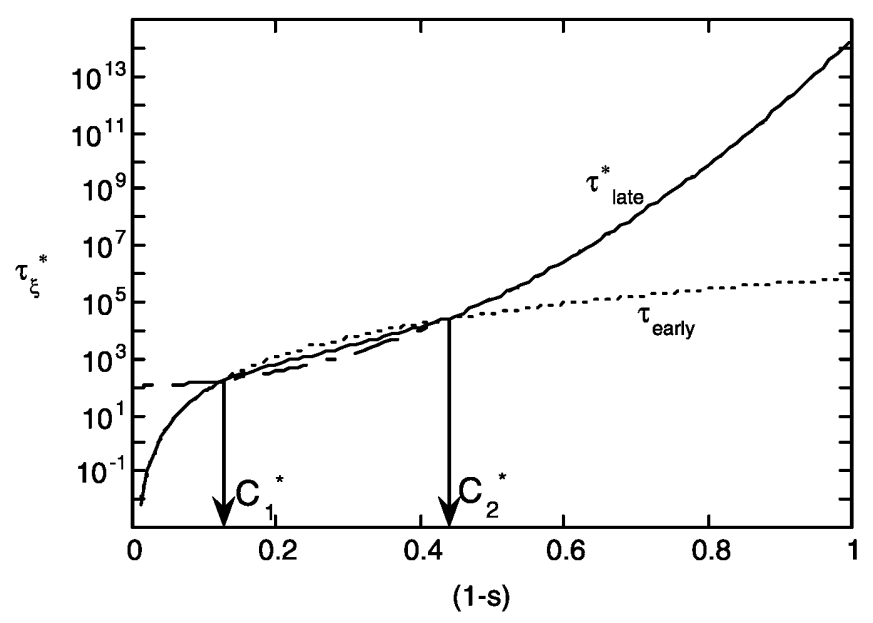

Fig. 1 The crossover function (solid line) from $\tau_{\text {early }}$ (dotted line) to $\left(\tau^{*}\right)$ (dashed line) with no constraint release $\left(\phi^{*}=1\right)$ for a linear polymer with 30 entanglements per chain or for a star with 15 entanglements per arm. The crossover points are indicated by $\mathrm{C}_{1}^{*}$ and $\mathrm{C}_{2}^{*}$. The $\mathrm{x}$-axis $(1-\mathrm{s})$ is the distance from the chain end $(1-\mathrm{s}=0)$ toward the branch point of the star or the center of the linear chain $(1-\mathrm{s}=1)$ 
release Rouse process has been given in Viovy et al. (1991), and Milner (1996). Here, we will merely adopt the formula from Milner and McLeish (1998) that constraint-release Rouse relaxation permits relaxation no faster with time than

$\psi_{\mathrm{R}}(t)=\psi^{*}\left(t_{0}\right)\left(\frac{t}{t_{0}}\right)^{-1 / 2}$

where $t_{0}$ is the time when $\psi^{*}(t)$ starts to drop faster than $t^{-1 / 2}$.

At time near zero, when the majority of the entanglement constraints still exist, the average survival probability of the chains $\phi_{(t)}^{*}$ equals $\psi_{(t)}^{*}$ and remains so until some time $t=t_{0}$, at which $\phi_{(t)}^{*}$ switches to $\psi_{\mathrm{R}}(t)$. At some later time $t$, if $\psi_{\mathrm{R}}(t)$ drops below $\psi_{(t)}^{*}, \phi_{(t)}^{*}$ then switches back to $\psi^{*}(t)$. Whenever $\psi^{*}(t)$ drops faster than $t^{-1 / 2}$ again, the constraint-release Rouse process will be activated and $\psi_{(t)}^{\mathrm{R}}$ from Eq. (7) with a new $\mathrm{t}_{0}$ will be used for $\phi_{(t)}^{*}$. The expression for $\phi^{*}(t)$ can therefore be written as

$\phi^{*}(t)=\psi^{*}(t) \quad$ when $\psi^{*}(t)>\psi_{\mathrm{R}}(t)$

$\phi^{*}(t)=\psi_{R}(t) \quad$ when $\psi^{*}(t)<\psi_{\mathrm{R}}(t)$

The average survival probability of the chains $\phi^{*}(t)$ will be used as the dilution factor in the fluctuation term in part B below.

\section{B. Reptation and fluctuation with constraint release}

In general, constraint-release manifests itself both in a local relaxation of an entanglement constraint and in a global loosening of the entanglement network. These modes of constraint release are called "tube reorganization" and "tube dilution," respectively (Viovy et al. 1991). While the local relaxation of entanglement constraints will be combined later in the final relaxation modulus, the time-dependent loosening of the entanglement network or tube widening, known as "dynamic dilution" (Ball and McLeish 1989), is included here in the activated fluctuation term.

Dynamic dilution takes into account the large differences in timescales between fast and slow relaxation processes by allowing the accumulation of constraint-release events to reduce the activation energy for deep-chain fluctuations. This reduction results in faster relaxation of the test chain. The dynamic-dilution mechanism is incorporated into the dual constraint model by using the reduced activation energy, $\mathrm{U}_{\text {eff }}\left(s_{\mathrm{i}}, t\right)$, instead of $\mathrm{U}^{*}\left(s_{i}\right)$, to calculate $\tau_{\text {late }, i}$. While this makes $\tau_{\text {late }, i}$ time dependent, $\tau_{\text {early }, i}$ remains the same as in part A. The expression for $\tau_{\text {early }, i}$ and $\tau_{\text {late }, i}$ with dynamic dilution can be written as (Mead, Van Dyke et al. 2000)

$$
\begin{aligned}
\tau_{\text {early }, i}\left(s_{i}\right)= & \frac{225}{256} \pi^{3} \frac{\tau_{\mathrm{R}, i}}{\mathrm{c}^{2}}\left(1-\mathrm{cs}_{i}\right)^{4}\left(\frac{\mathrm{N}_{\mathrm{en}, i}}{\mathrm{c}}\right)^{2} \\
& \text { and } \tau_{\text {late }, i}\left(s_{i}, t\right)=\frac{\tau_{\mathrm{R}, i}}{\mathrm{c}^{2}} \exp \left(\mathrm{U}_{\mathrm{eff}}\left(s_{i}, \mathrm{t}\right)\right)
\end{aligned}
$$

where $\mathrm{U}_{\text {eff }}\left(s_{i}, t\right)$ is obtained by multiplying $\mathrm{U}^{*}$ by the dilution factor $\phi^{*}(t)$ calculated from Eq. (8) giving

$\mathrm{U}_{\mathrm{eff}}\left(s_{i}, t\right)=\frac{15}{8} \frac{\mathrm{N}_{\mathrm{en}, i}}{\mathrm{c}} \phi^{*}(t)\left(1-\mathrm{cs}_{i}\right)^{2}$

As shown in Fig. 2, although $\tau_{\zeta, \mathrm{i}}$ has behavior similar to that in part $\mathrm{A}$, the crossover locations, $\mathrm{C}_{1}$ and $\mathrm{C}_{2}$, are now changing with time due to the time dependence of $\tau_{\text {late, }, \text {. Hence, }} \tau_{\zeta, \text { i }}$ along the tube position $s_{i}$ is described by Eq. (4) with $\tau_{\text {late }, i}^{*}$ replaced by $\tau_{\text {late }, i}$ :

$$
\begin{aligned}
\tau_{\xi, i} & =\tau_{\text {early }, i} & & \text { when }\left(1-s_{i}\right)<\mathrm{C}_{1} \\
\tau_{\xi, i} & =\sqrt{\tau_{\text {early }, i} \tau_{\text {late }, i}} & & \text { when } \mathrm{C}_{1}<\left(1-s_{i}\right)<\mathrm{C}_{2} \\
\tau_{\xi, i} & =\tau_{\text {late }, i} & & \text { when }\left(1-s_{i}\right)>\mathrm{C}_{2}
\end{aligned}
$$

where $\mathrm{C}_{1}$ and $\mathrm{C}_{2}$ are again the crossover points.

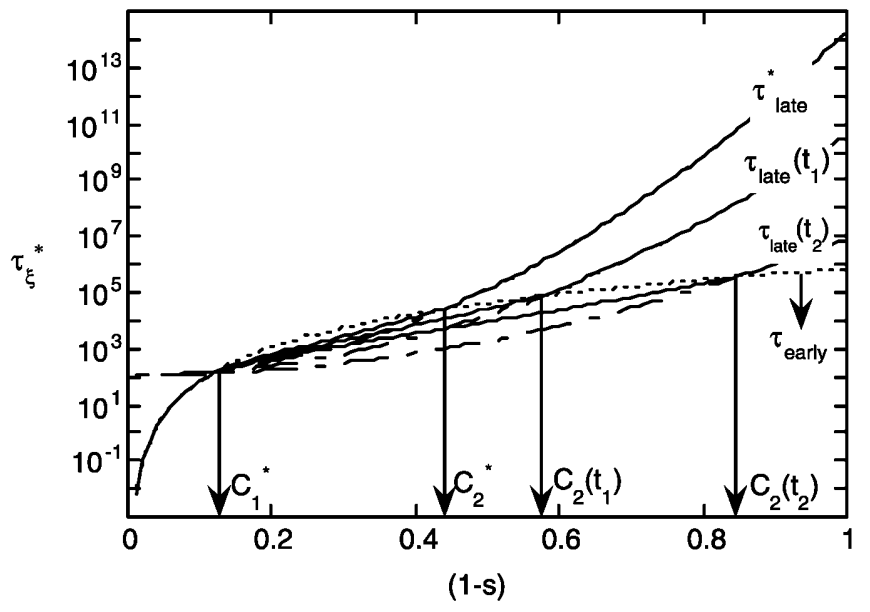

Fig. 2 The crossover function from $\tau_{\text {early }}$ to $\tau_{\text {late }}$ with constraint release $\left(\phi^{*}(t)=1.0,0.7\right.$, and 0.4$)$ for the polymer of Fig. 1. The crossover points $\mathrm{C}_{1}$ and $\mathrm{C}_{2}$ change with $\phi^{*}(t)$, which decreases with time

This new expression for $\tau_{\xi, i}$ is now incorporated into the diffusion equation as follows:

$\frac{\partial p_{i}\left(s_{i}, t\right)}{\partial \mathrm{t}}=\frac{D_{i}}{L_{i}^{2}} \frac{\partial^{2} p_{i}\left(s_{i}, t\right)}{\partial s_{i}^{2}}-\frac{p_{i}\left(s_{i}, t\right)}{\tau_{\xi, i}\left(s_{i}\right)}$

where $p_{i}\left(s_{i}, t\right)$ and $\tau_{\xi, i}$ are the tube segment survival probability and the time constant for contour length fluctuation, respectively, in the presence of constraint release by dynamic dilution (Eq. 11).

The overall survival probability $\phi_{i}(t)$ of a tube occupied by chain $i$ can then be calculated from $p_{i}\left(s_{i}, t\right)$ by

$\phi_{i}(t)=\int_{0}^{1} p_{i}\left(s_{i}, t\right) \mathrm{d} s_{i}$

and the average survival probability of all tubes is

$\phi(t)=\sum w_{i} \phi_{i}(t)$

$\phi(t)$ can again be obtained numerically by solving for $p_{i}\left(s_{i}, t\right)$ using the Crank-Nicolson method, (Using logarithmic time) then converting to $\phi_{i}(t)$ and $\phi(t)$ by means of Eqs. (13) and (14), respectively.

So far, we have only accounted for "global" constraint-release effects that accelerate contour-length fluctuations via the dilution factor $\phi^{*}(t)$, which is used in the fluctuation potential $\mathrm{U}_{\text {eff }}$. We can describe local constraint-release events using the double-diffusion mechanism, developed as an extension of double reptation (Tsenoglou 1987; des Cloizeaux 1988) by Mead, Van Dyke et al. (2000). This theory considers the survival probability of a binary topological interaction of a test chain with a surrounding chain. This mechanism accounts for the effect of the surrounding chains on the overall relaxation process by multiplying the average survival probability of the test chain by that of the constraints surrounding the chain. Without the constraint-release Rouse process, the multiplication would be $\phi^{2}(t)$. However, after including constraint-release Rouse processes, the fraction of constraints $\phi^{\prime}(t)$ that block motion of a test chain at time $t$ can differ from the fraction of tube segments $\phi(t)$ that are still occupied at time $t$. Thus

$\phi^{\prime}(t)=\phi(t) \quad$ when $\phi(t)<\phi_{\mathrm{R}}(t)$

$\phi^{\prime}(t)=\phi_{\mathrm{R}}(t) \quad$ when $\phi(t)<\phi_{\mathrm{R}}(t)$ 
where $\phi_{\mathrm{R}}(t)=\phi\left(t_{0}\right)\left(\frac{t}{t_{0}}\right)^{-1 / 2}, t_{0}$ is the time when $\phi(t)$ begins to drop faster than $t^{-1 / 2}$, and $\phi\left(t_{0}\right)$ is the average survival probability at $t_{0}$. The activation of constraint-release Rouse processes for the fraction of local constraints $\phi^{\prime}(t)$ in Eq. (15) is identical to that for the dilution term $\phi^{*}(t)$ in Eq. (8). That is, at time near zero, $\phi^{\prime}(t)$ equals $\phi(t)$. After that, at each time t, $\phi^{\prime}(t)$ is assigned the greater of the values of $\phi(t)$ and $\phi_{\mathrm{R}}(t)$.

Thus, the overall survival probability of the binary interactions between the test chains and the surrounding chains becomes

$\Phi(t)=\phi(t) \phi^{\prime}(t)$

The relaxation modulus $\mathrm{G}(t)$ from the contributions of reptation, contour-length fluctuations, and constraint release can be obtained by multiplying $\Phi(t)$ by the plateau modulus $\mathrm{G}_{\mathrm{N}}^{0}$ :

$\mathrm{G}(t)=\mathrm{G}_{\mathrm{N}}^{0} \Phi(t)=\mathrm{G}_{\mathrm{N}}^{0} \phi(t) \phi^{\prime}(t)$

The final relaxation mechanism we consider is high-frequency Rouse relaxation within the tube (not to be confused with the constraint-release Rouse processes by which a chain escapes one tube and explores a wider one). High-frequency Rouse relaxation includes stress relaxations of segments of the test chain at timescales both shorter than $\tau_{\mathrm{e}}$, the time at which the chain segments first feel the constraint imposed by the conceptual tube, and longer than $\tau_{\mathrm{e}}$, during which only the longitudinal Rouse modes along the tube are available. The "equilibration time" $\tau_{\mathrm{e}}$ is the Rouse relaxation time for an entanglement segment of a chain and is independent of chain length, i.e., the molecular weight of the polymer. $\tau_{\mathrm{e}}$ is related to $\tau_{\mathrm{R}, \mathrm{i}}$ by $\tau_{e}=2 \tau_{\mathrm{R}, i} / \mathrm{N}_{\mathrm{en}, i}^{2}$ (Doi and Edwards 1986, p 214), which implies that $\tau_{\mathrm{e}}$ is related to $\tau_{\mathrm{d}, \mathrm{i}}$ as $\tau_{e}=\tau_{\mathrm{d}, i} / 3 \mathrm{~N}_{\mathrm{en}, i}^{3}$. The division of spatial scales separating Rouse processes with time-scales less than $\tau_{\mathrm{e}}$ from those with timescales greater than $\tau_{\mathrm{e}}$ has been represented by Milner and McLeish (1998) using an approximate "fragmented Rouse" spectrum to calculate the Rouse relaxation modulus of chain $i$ $\left(\mathrm{G}_{\mathrm{R}, i}\right)$ :

$\mathrm{G}_{\mathrm{R}, i}(t)=\frac{1}{3} \mathrm{G}_{\mathrm{N}}^{0} \sum_{\mathrm{k}=1}^{\mathrm{N}_{\mathrm{en}, i}} \exp \left(\frac{-\mathrm{k}^{2} t}{\tau_{\mathrm{R}, i}}\right)+\mathrm{G}_{\mathrm{N}}^{0} \sum_{\mathrm{k}=\mathrm{N}_{\mathrm{en}, i}}^{\mathrm{N}} \exp \left(\frac{-\mathrm{k}^{2} t}{\tau_{\mathrm{R}, i}}\right)$

Here, the first term accounts for the slow longitudinal modes which are confined by the tube to one dimension rather than three as in the ordinary Rouse theory; hence, their magnitude is reduced by a factor of three. The second term represents fast three-dimensional relaxation of portions of the chain within a single tube segment.

Finally, the full stress-relaxation modulus $\left(\mathrm{G}_{\text {total }}\right)$ combines $\mathrm{G}(t)$ from Eq. 17 with the contribution from Rouse processes giving

$\mathrm{G}_{\mathrm{total}}(\mathrm{t})=\mathrm{G}(\mathrm{t})+\sum_{i} w_{i} \mathrm{G}_{\mathrm{R}, i}(t)$

The stress-relaxation modulus can then be converted to the storage modulus $\left(\mathrm{G}^{\prime}\right)$ and the loss modulus $\left(\mathrm{G}^{\prime \prime}\right)$ by

$\mathrm{G}^{\prime}(\omega)=\omega \int_{0}^{\infty} \mathrm{G}_{\text {total }}(t) \cos (\omega \mathrm{t}) \mathrm{d} t$, and
$\mathrm{G}^{\prime \prime}(\omega)=\omega \int_{0}^{\infty} \mathrm{G}_{\text {total }}(t) \sin (\omega \mathrm{t}) \mathrm{d} t$

The calculated $G^{\prime}$ and $G^{\prime \prime}$ can be compared with experimental data for polybutadiene, polyisoprene, and polystyrene, for both linear and star architectures. The comparison for monodisperse, bidisperse, and polydisperse polymers are presented in the following section.

\section{Results and discussion}

For well entangled linear polymers, the longest relaxation time, which is related to reptation, would be proportional to $\mathrm{M}^{3}$ if reptation were the only relaxation process. However, since other relaxation processes are also active near the terminal region, the terminal relaxation time in the experiments always shows a dependence on molecular weight with a power-law exponent higher than 3. For the typical range of polymer molecular weights, the terminal relaxation time is found to be proportional to $\mathrm{M}^{3.4}$. Comparison of the dual constraint model with experiments shows that double diffusion and dynamic dilution improve the linear viscoelastic predictions at low and intermediate frequencies. While the double diffusion improves the predictions especially in the terminal region, the early fast diffusion, which is included in the contour-length fluctuation mechanism, improves the prediction of $\mathrm{G}^{\prime \prime}$ by attenuating the steep decrease in the intermediatefrequency region (Milner and McLeish 1997). For star polymers, the relaxation mechanisms are the same as for linear polymers except that star polymers do not reptate; therefore, the most important relaxation mechanism for star polymers is contour-length fluctuation. At high frequencies, the Rouse relaxation processes create upturns in $G^{\prime}$ and $G^{\prime \prime}$ for both linears and stars.

The parameters used in the model are the plateau modulus $\left(\mathrm{G}_{\mathrm{N}}^{0}\right)$, the relaxation time for a segment of a chain $\left(\tau_{\mathrm{e}}\right)$, and the polymer molecular weight or the number of entanglements per polymer chain $\left(N_{e n}=M / M_{e}\right) . \quad M_{e}$ is the molecular weight between two entanglements and can be computed from the value of $\mathrm{G}_{\mathrm{N}}^{0}$ by $\mathrm{M}_{\mathrm{e}}=\rho \mathrm{k}_{\mathrm{B}} \mathrm{TN}_{\mathrm{A}} / \mathrm{G}_{\mathrm{N}}^{0}$ where $\mathrm{N}_{\mathrm{A}}$ is Avogadro's number, $\mathrm{T}$ is the absolute temperature, $\mathrm{k}_{\mathrm{B}}$ is Boltzmann's constant, and $\rho$ is the density of the polymer (Ferry 1980). Since $\tau_{\mathrm{d}, i}=\left(\zeta \mathrm{N}_{i}^{3} \mathrm{~b}^{4}\right) /\left(\pi^{2} \mathrm{k}_{\mathrm{b}} \mathrm{T} a^{2}\right)$ and $\tau_{\mathrm{e}}=$ $\tau_{\mathrm{d}, i} / 3 \mathrm{~N}_{\mathrm{en}, \mathrm{i}}^{3}$ as mentioned earlier, $\tau_{\mathrm{e}}$ can be related to the monomeric friction coefficient $\zeta$ and the tube diameter $a$ by $\tau_{\mathrm{e}}=\zeta a^{2} \mathrm{~N}_{i} / 3 \pi^{2} \mathrm{~N}_{\mathrm{en}, \mathrm{i}} \mathrm{k}_{\mathrm{B}} \mathrm{T}$. By substituting $\mathrm{N}_{i}=\mathrm{M}_{i} / \mathrm{M}_{0}$ and $\mathrm{N}_{\mathrm{en}, \mathrm{i}}=\mathrm{M}_{i} / \mathrm{M}_{\mathrm{e}}, \tau_{\mathrm{e}}$ can be rewritten as $\zeta a^{2} \mathrm{M}_{\mathrm{e}} / 3 \pi^{2} \mathrm{k}_{\mathrm{B}} \mathrm{TM}_{0}$. Although $\tau_{\mathrm{e}}$ is calculated from $\zeta$ and $a$, the tube diameter $a$ reported in the literature is calculated from $\mathrm{G}_{\mathrm{N}}^{0}$, leaving a direct proportion of $\tau_{\mathrm{e}}$ and $\zeta$. As can be seen from this relationship, $\tau_{\mathrm{e}}$ is independent of polymer molecular weight and architecture; therefore, we can correlate the behavior of linear and star polymers of the same chemical composition by using the same $\tau_{\mathrm{e}}$ and $\mathrm{G}_{\mathrm{N}}^{0}$ for both architectures. Of these, $G_{N}^{0}$ is essentially temperature-independent, while $\tau_{\mathrm{e}}($ or $\zeta$ ) is very sensitive to temperature. These two model parameters are taken from literature, where available. When the value of $\zeta$ is unavailable, which is the case for polyisoprene and polystyrene at low temperatures, we obtain $\tau_{\mathrm{e}}$ by fitting the model to 
experimental data for a linear polymer, and consistently using the value obtained for all other linear and star polymers having the same chemical composition at the same temperature. The model parameters for all polymers tested in this paper are summarized in Table 1.

The comparisons of the predictions with experiments are reported for monodisperse, bidisperse, and polydisperse polymers. In the monodisperse case, we find that for high molecular weight polymers, the model predicts the experimental data reasonably well using literature values of $\tau_{\mathrm{e}}$ and $\mathrm{G}_{\mathrm{N}}^{0}$, with no fitting parameters. However, the predictions at low numbers of entanglements $\mathrm{N}_{\mathrm{en}}$ are not very accurate. This problem might be expected since the "tube" concept becomes questionable for a low number of entanglements. In order to identify the experimental data in which the model predictions might be expected to fail, we use open symbols $(\mu)$ to indicate experimental data that have $\mathrm{N}_{\mathrm{en}}$ less than 10 . For bidisperse polymers, the linearlinear blend and star-star blend behaviors are predicted reasonably well for the limited experimental data reported. For polymers with continuous polydispersity, comparisons between the model predictions and the experiments are reported for 2 sets of polystyrene consisting of mixtures of 11 and 13 components of linear monodisperse polymers of different molecular weight. We found that the model can capture accurately the effect of very small amounts of long-chain components. Case studies for monodisperse, bidisperse, and polydisperse polymers are described subsequently in separate sections for several linear and star polymers.

Monodisperse polymers - case studies

\section{Polybutadiene (PBD)}

Linear $P B D$. The experimental data for linear polybutadienes at $28^{\circ} \mathrm{C}$ were taken from Baumgaertel et al.
(1992) who reported $G^{\prime}$ and $G^{\prime \prime}$ plots for four different molecular weights $(\mathrm{M}): 20.7 \mathrm{~K}, 44.1 \mathrm{~K}, 97 \mathrm{~K}$, and $201 \mathrm{~K}$, where the " $\mathrm{K}$ " represents a factor of 1000 . The polydispersity index (P.I. $=\mathrm{M}_{\mathrm{w}} / \mathrm{M}_{\mathrm{n}}$ ) for all of these polymers is less than 1.10 except for the sample of highest molecular weight, which has a P.I. of 1.27. For the model calculations, the molecular weight between entanglements $\left(\mathrm{M}_{\mathrm{e}}\right)$ is calculated from $\mathrm{G}_{\mathrm{N}}^{0}$ by $\mathrm{M}_{\mathrm{e}}=\rho \mathrm{k}_{\mathrm{b}} \mathrm{TN}_{\mathrm{A}} / \mathrm{G}_{\mathrm{N}}^{0}$ (Ferry 1980). The parameters $\mathrm{G}_{\mathrm{N}}^{0}$, $\mathrm{M}_{\mathrm{e}}$, and $a$ for PBD are found from Fetters et al. (1994) to be $1.25 \times 10^{6} \mathrm{~Pa}, 1815$, and $44.4 \times 10^{-8} \mathrm{~cm}$, respectively. Since Fetters' value of $M_{e}$ is based on the equation $\mathrm{M}_{\mathrm{e}}=\frac{4}{5} \rho \mathrm{k}_{\mathrm{b}} \mathrm{TN}_{\mathrm{A}} / \mathrm{G}_{\mathrm{N}}^{0}$, which contains a factor of $4 / 5$ that is not present in the definition of $M_{e}$ used by Ferry, we must multiply Fetters' values of $M_{e}$ by $5 / 4$, giving $M_{e}=2269$ (Ferry's definition). Consequently, the tube diameter, $a$, which is given by $a=\left(\mathrm{b} \sqrt{\mathrm{M}_{\mathrm{e}} / \mathrm{M}}\right)$, must be increased by a factor of $\sqrt{5 / 4}$, giving $a=49.6 \times 10^{-8} \mathrm{~cm}$. The corresponding values of $\mathrm{N}_{\mathrm{en}} \equiv \mathrm{M} / \mathrm{M}_{\mathrm{e}}$ for the four samples are 9.12, 19.44, 42.75 , and 88.59 , respectively. The polymers are assumed to be monodisperse for all calculations except for the highest molecular-weight sample (which has the highest polydispersity). For this sample, we account for the distribution of molecular weights in our calculations by discretizing the molecular weights into 20 components with the weight fractions corresponding to the area under the molecular-weight-distribution curve. The monomeric friction coefficient $(\zeta)$ is taken from Ferry (1980, p 331) to be $10^{-6.75} \mathrm{~g} / \mathrm{s}$ at $25^{\circ} \mathrm{C}$. This is shifted slightly using the WLF equation (Ferry 1980, p 227) to $10^{-6.8078} \mathrm{~g} / \mathrm{s}$ at the test temperature of $28^{\circ} \mathrm{C}$. From $\zeta$ and $a$, the corresponding value of $\tau_{\mathrm{e}}$ for polybutadiene at $28{ }^{\circ} \mathrm{C}$ turns out to be $1.31 \times 10^{-6} \mathrm{~s}$. The comparison between theory and experiments for linear PBD is shown in Fig. 3a, b. As can be seen, the model predicts the experimental data well.
Table 1 Summary of the model parameters for polybutadienes, polystyrene, and polyisoprene

\begin{tabular}{|c|c|c|c|c|c|}
\hline Polymer & $\zeta\left(\mathrm{g} \mathrm{s}^{-1}\right)$ & $\mathrm{G}_{\mathrm{N}}^{0}(\mathrm{~Pa})^{\mathrm{b}}$ & $\begin{array}{l}\mathrm{M}_{\mathrm{e}} \\
\left(\mathrm{g} \mathrm{mol}^{-1}\right)^{\mathrm{c}}\end{array}$ & $a(\mathrm{~cm})^{\mathrm{c}}$ & $\tau_{\mathrm{e}}(\mathrm{s})$ \\
\hline \multicolumn{6}{|l|}{ PBD } \\
\hline$\left(25^{\circ} \mathrm{C}\right)$ & $10^{-6.75}$ & $25 \times 10^{6}$ & 2,269 & $49.6 \times 10^{-8}$ & $1.51 \times 10^{-6}$ \\
\hline$\left(28^{\circ} \mathrm{C}\right)$ & & & & & ${ }^{\mathrm{a}} 1.31 \times 10^{-6}$ \\
\hline$\left(30^{\circ} \mathrm{C}\right)$ & & & & & ${ }^{\mathrm{a}} 9.315 \times 10^{-7}$ \\
\hline \multicolumn{6}{|l|}{ PS } \\
\hline$\left(150^{\circ} \mathrm{C}\right)$ & $\mathrm{n} / \mathrm{c}$ & $2 \times 10^{5}$ & 16,625 & $85.5 \times 10^{-8}$ & $5 \times 10^{-2}$ \\
\hline$\left(160^{\circ} \mathrm{C}\right)$ & $\mathrm{n} / \mathrm{c}$ & & & & $1 \times 10^{-2}$ \\
\hline$\left(169.5^{\circ} \mathrm{C}\right)$ & $3.02 \times 10^{-5}$ & & & & $1.96 \times 10^{-3}$ \\
\hline$\left(180{ }^{\circ} \mathrm{C}\right)$ & $9.85 \times 10^{-6}$ & & & & $6.22 \times 10^{-4}$ \\
\hline \\
\hline$\left(25^{\circ} \mathrm{C}\right)$ & $\mathrm{n} / \mathrm{a}$ & $4.34 \times 10^{5}$ & 5200 & $62 \times 10^{-8}$ & $6 \times 10^{-5}$ \\
\hline$\left(75^{\circ} \mathrm{C}\right)$ & $\mathrm{n} / \mathrm{a}$ & & & & $4 \times 10^{-5}$ \\
\hline
\end{tabular}

$\mathrm{n} / \mathrm{c}=$ data not accurate, $\mathrm{n} / \mathrm{a}=$ data not available

${ }^{a}$ Data obtained from WLF shifting from $25^{\circ} \mathrm{C}$

${ }^{\mathrm{b}}$ Data taken from Fetters et al. 1994

${ }^{\mathrm{c}}$ Using Ferry's definition i.e., $\mathrm{M}_{\mathrm{e}}=\rho \mathrm{k}_{\mathrm{b}} \mathrm{TN}_{\mathrm{A}} / \mathrm{G}_{\mathrm{N}}^{0}$ 


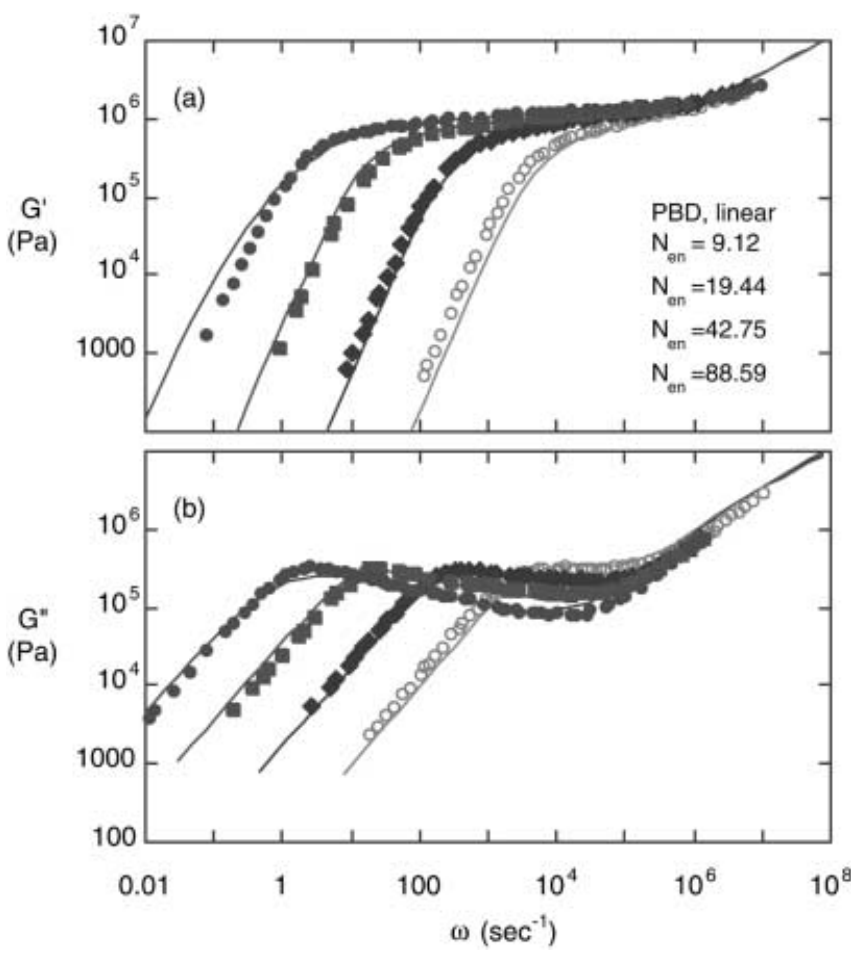

Fig. 3a, b Comparison of the model predictions of: a storage modulus, $\mathrm{G}^{\prime}$; b loss modulus, $\mathrm{G}^{\prime \prime}$, with experimental data for linear polybutadienes at $28{ }^{\circ} \mathrm{C}$ (Baumgaertel et al. 1992). The molecular weights of the samples from right to left are $20.7 \mathrm{~K}, 44.1 \mathrm{~K}, 97 \mathrm{~K}$, and $201 \mathrm{~K}$, respectively. The solid lines are the model predictions using the literature values of the parameters: $\mathrm{G}_{\mathrm{N}}^{0}=1.25 \times 10^{6} \mathrm{~Pa}$ and $\tau_{\mathrm{e}}=1.31 \times 10^{-6} \mathrm{~s}$. In this and subsequent figures, the open symbols represent data for polymers with less than $\mathrm{N}_{\mathrm{en}}=10$ entanglements per chain or per branch

Star PBD. The experimental data for star polybutadienes at $25^{\circ} \mathrm{C}$ were taken from Adams et al. (1996). The linear viscoelastic data were reported for four samples having different number of arms and almost the same number of entanglements per arm, each with a very narrow molecular weight distribution. The molecular weights of all samples with the numbers of arms, functionalities $(f)$, and amounts of residual unreacted linear chains $(l)$ are summarized in Table 2.

We use the model parameters $\mathrm{G}_{\mathrm{N}}^{0}=1.25 \times 10^{6} \mathrm{~Pa}$, $\mathrm{M}_{\mathrm{e}}=2269$, and $a=49.6 \times 10^{-8} \mathrm{~cm}$, the same as for

Table 2 Functionality, molecular weight $\left(\mathrm{g} \mathrm{mol}^{-1}\right)$, fraction of residual linear chains, and the diluted number of entanglement per arm of PBD star-branched polymers

\begin{tabular}{rrlll}
\hline Star PBD & $f$ & $\mathrm{M}_{\mathrm{W}}$ & $l(\%)$ & $\begin{array}{l}\mathrm{N}_{\text {en }} \\
\text { (diluted) }\end{array}$ \\
\hline $3 \mathrm{arms}$ & 2.8 & 101,600 & 4.3 & 15.3 \\
$4 \mathrm{arms}$ & 3.9 & 145,700 & 7.0 & 15.3 \\
$8 \mathrm{arms}$ & 7.2 & 240,900 & 9.7 & 13.3 \\
$12 \mathrm{arms}$ & 11.8 & 369,300 & 4.5 & 13.18 \\
\hline
\end{tabular}

linear PBD. The monomeric friction coefficient $(\zeta)$ from Ferry is $10^{-6.75} \mathrm{~g} / \mathrm{s}$ at $25^{\circ} \mathrm{C}$ for PBD (Ferry 1980 , p 331). $\tau_{\mathrm{e}}$ associated with these values of $\zeta$ and $a$ for PBD is $1.51 \times 10^{-6} \mathrm{~s}$. The corresponding numbers of entanglements per arm $\left(\mathrm{N}_{\mathrm{en}}\right)$ for these samples are 15.99, 16.47, 14.75 , and 13.79 , respectively. The small fraction of linear chains in the polymer samples act like a solvent; thus diluting $\mathrm{N}_{\mathrm{en}}$ of the star polymers in each sample proportionally to the amount of linear chains present. Therefore, $\mathrm{N}_{\mathrm{en}}$ turn out to be 15.3,15.3, 13.3, and 13.18 for star polymers with $3 \mathrm{arms}, 4 \mathrm{arms}, 8 \mathrm{arms}$, and 12 arms, respectively, as reported in Table 2. The model predicts no dependence of the rheological properties of stars on the number of arms. As shown in Fig. $4 a-d$, the model for star PBD agrees fairly well with all experimental data, especially for 4-arm and 8-arm stars (Fig. 4b, c). However, the prediction for the 3arm star (Fig. 4a) slightly overestimates the experimental relaxation time; on the other hand, the experimental relaxation time is underestimated for the 12-arm star. This implies a certain degree of unexplained armnumber dependence of the rheological properties of star polymers. The discrepancy for the 3-arm star polymers is thought to be due to the diffusion of the branch point of the star in the direction of the arms (Klein 1986) causing a faster than predicted relaxation of the polymers.

\section{Polystyrene (PS)}

Linear PS. There are two sources of experimental data for linear polystyrene with narrow molecular weight distributions. One is for samples of molecular weight $290 \mathrm{~K}, 750 \mathrm{~K}$, and $3000 \mathrm{~K}$ at $180{ }^{\circ} \mathrm{C}$ (Schausberger et al. 1985); the other set is for molecular weights (weight average) of $275 \mathrm{~K}$ and $860 \mathrm{~K}$ at $169.5^{\circ} \mathrm{C}$ (Graessley and Roover 1979). The model parameters for PS are $\mathrm{G}_{\mathrm{N}}^{0}=2 \times 10^{5} \mathrm{~Pa}, \mathrm{M}_{\mathrm{e}}=16,625$, and $a=85.5 \times$ $10^{-8} \mathrm{~cm}$, (all are from Fetters et al. 1994, with $\mathrm{M}_{\mathrm{e}}$ and $a$ corrected by the factors of $5 / 4$ and $\sqrt{5 / 4}$, respectively, as earlier). With $\mathrm{M}_{\mathrm{e}}=16625$, those molecular weights of PS at $180{ }^{\circ} \mathrm{C}$ correspond to $\mathrm{N}_{\mathrm{en}}=17.44,45.11$, and 180.45 , and for PS at $169.5^{\circ} \mathrm{C}$; the corresponding $\mathrm{N}_{\mathrm{en}}$ are 16.54 and 51.73. The monomeric friction coefficient, $\zeta$, is calculated from the experimental correlation $\zeta=\zeta_{\infty} \mathrm{e}^{\frac{\mathrm{B}}{(\mathrm{T}-49.6)}}$ where $\mathrm{T}$ is the testing temperature in ${ }^{\circ} \mathrm{C}$, $\zeta_{\infty}=2.7 \times 10^{-11} \mathrm{~g} / \mathrm{s}$ for high molecular-weight polystyrenes, and the constant $\frac{\mathrm{B}}{\alpha}$ equals $1620 \pm 50$ (Majeste et al. 1998). Here, we use the upper bound, which is $\frac{\mathrm{B}}{\alpha}=1670$. The values of $\zeta$ are then $9.85 \times 10^{-6} \mathrm{~g} / \mathrm{s}$ at $1^{\alpha} 80{ }^{\circ} \mathrm{C}$ and $3.02 \times 10^{-5} \mathrm{~g} / \mathrm{s}$ at $169.5^{\circ} \mathrm{C}$. Thus, $\tau_{\mathrm{e}}$ for PS is $6.22 \times 10^{-4} \mathrm{~s}$ at $180{ }^{\circ} \mathrm{C}$ and $1.955 \times 10^{-3} \mathrm{~s}$ at $169.5^{\circ} \mathrm{C}$. The comparisons of the model predictions with experiments are shown in Fig. 5a, b for the experimental data set at $180{ }^{\circ} \mathrm{C}$ and in Fig. 6a, b for 

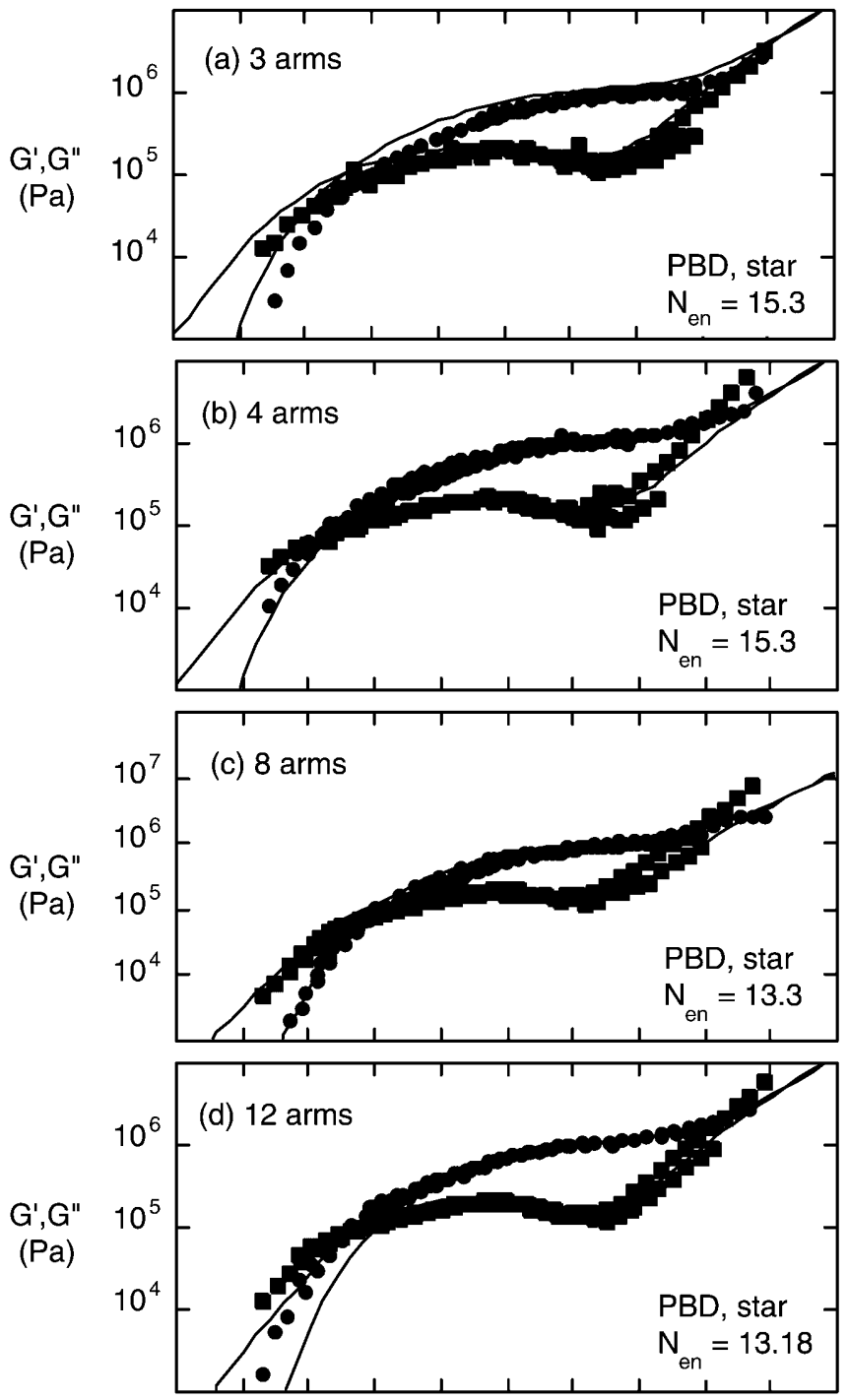

$\omega\left(\sec ^{-1}\right)$

Fig. 4a-d Comparison of the model predictions of storage modulus, $\mathrm{G}^{\prime}$, and loss modulus, $\mathrm{G}^{\prime \prime}$, with experimental data for star polybutadienes at $25^{\circ} \mathrm{C}$ (Adams et al. 1996). The molecular weights and functionalities $(f)$ of the stars are: a $\mathrm{MW}=97.2 \mathrm{~K}(f=2.8)$; b $\mathrm{MW}=135.5 \mathrm{~K}(f=3.9)$; $\mathbf{M W}=217.5 \mathrm{~K}(f=7.2)$; $\mathbf{d} \mathrm{MW}=353 \mathrm{~K}$ $(f=11.8)$. The solid lines are the model predictions using the literature values of the parameters: $\mathrm{G}_{\mathrm{N}}^{0}=1.25 \times 10^{6} \mathrm{~Pa}$ and $\tau_{\mathrm{e}}=1.51 \times 10^{-6} \mathrm{~s}$

the one at $169.5^{\circ} \mathrm{C}$. The predictions agree well with all experimental data except for the lowest $\mathrm{N}_{\mathrm{en}}$ at $180{ }^{\circ} \mathrm{C}$ $\left(\mathrm{N}_{\mathrm{en}}=17.44\right)$.

Star PS. The experimental data for PS stars (Graessley and Roover 1979) are for the samples with molecular weights of $1027 \mathrm{~K}$ (four arms) and $1090 \mathrm{~K}$ (six arms) at $169.5^{\circ} \mathrm{C}$. All model parameters for the PS stars are the same as the linear PS at $169.5^{\circ} \mathrm{C}$ (i.e., $\mathrm{G}_{\mathrm{N}}^{0}=2 \times 10^{5} \mathrm{~Pa}$,

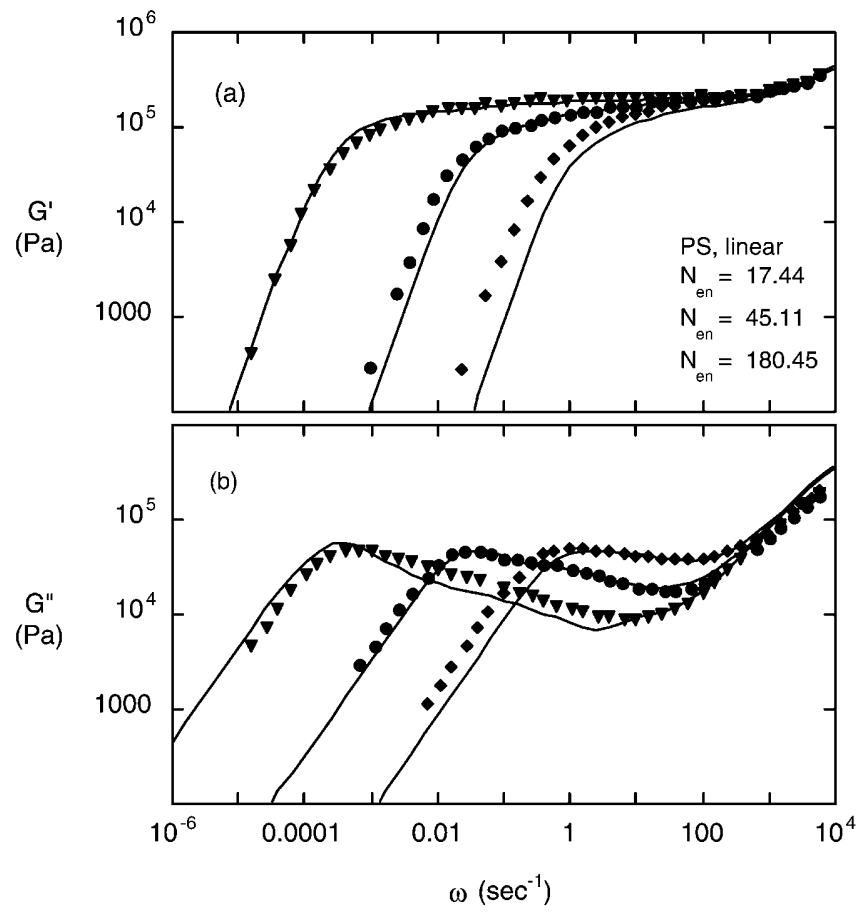

Fig. 5a, b Comparison of the model predictions of: a storage modulus $\left(\mathrm{G}^{\prime}\right)$; b loss modulus $\left(\mathrm{G}^{\prime \prime}\right)$ with experimental data for linear polystyrenes at a reference temperature of $180{ }^{\circ} \mathrm{C}$ (Schausberger et al. 1985). The arm molecular weights of the samples from right to left are $289.97 \mathrm{~K}, 750 \mathrm{~K}$, and $3000 \mathrm{~K}$, respectively. The solid lines are the model predictions using the literature values of the parameters: $\mathrm{G}_{\mathrm{N}}^{0}=2 \times 10^{5} \mathrm{~Pa}$ and $\tau_{\mathrm{e}}=6.22 \times 10^{-4} \mathrm{~s}$

$\mathrm{M}_{\mathrm{e}}=16625$, and $\left.\tau_{\mathrm{e}}=1.955 \times 10^{-3} \mathrm{~s}\right)$. The numbers of entanglements per arm for the PS stars are 15.44 and 10.93, respectively. As is shown in Fig. 7a, b, the model predictions agree fairly well with the experimental data for $\mathrm{N}_{\mathrm{en}}=15.44$. However, the model underestimates the longest relaxation time for the six-arm PS star with $\mathrm{N}_{\mathrm{en}}=10.93$ by a factor of 4 . This might be expected because of its small number of entanglements.

\section{Polyisoprene (PI)}

Linear PI. The experimental data from Fetters et al. (1993) are used for the comparisons for both linear and star polyisoprene. The linear polyisoprene studied by Fetters et al. has a molecular weight of $500 \mathrm{~K}\left(25^{\circ} \mathrm{C}\right)$. The parameters for PI are taken from Pearson et al. (1983) which give $\mathrm{M}_{\mathrm{e}}=5200$ and $\mathrm{G}_{\mathrm{N}}^{0}=4.34 \times 10^{5} \mathrm{~Pa}$ (Ferry's definition); thus, $\mathrm{N}_{\mathrm{en}}$ for this data is 96.15 . Since the literature value of $\zeta$ is unavailable for PI, $\tau_{\mathrm{e}}$ for this case is left as the only fitting parameter. The comparison between the predictions and the experimental data are shown in Fig. 8 using $\tau_{\mathrm{e}}=6.0 \times 10^{-5} \mathrm{~s}$. As can be seen, the model fits the experimental data well. 


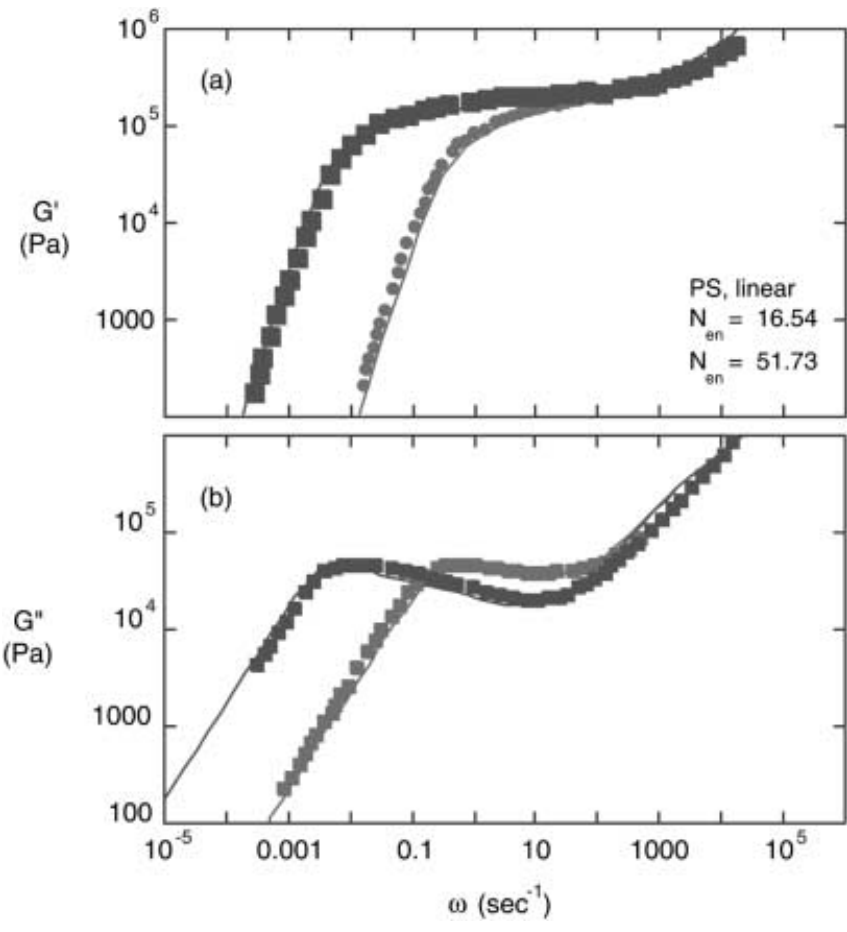

Fig. 6a, b Comparison of the model predictions of: a storage modulus $\left(G^{\prime}\right)$; b loss modulus $\left(G^{\prime \prime}\right)$ with experimental data for linear polystyrenes at a reference temperature of $169.5^{\circ} \mathrm{C}$ (Graessley and Roover 1979). The molecular weights of the samples from right to left are $275 \mathrm{~K}$ and $860 \mathrm{~K}$, respectively. The solid lines are the model predictions using the literature values of the parameters: $\mathrm{G}_{\mathrm{N}}^{0}=2 \times 10^{5} \mathrm{~Pa}$ and $\tau_{\mathrm{e}}=1.96 \times 10^{-3} \mathrm{~s}$

Star PI. The experimental data for polyisoprene 4-arm stars are also taken from Fetters et al. (1993). The data were reported for the arm molecular weights of $95 \mathrm{~K}$, $72.2 \mathrm{~K}$, and $47.5 \mathrm{~K}$ at $25{ }^{\circ} \mathrm{C}$ with narrow molecular weight distributions. Their experimental data allow the effect of arm length to be studied. We use the same values of the model parameters $\mathrm{G}_{\mathrm{N}}^{0}$ and $\mathrm{M}_{\mathrm{e}}$ as for linear PI. The corresponding values of $\mathrm{N}_{\mathrm{en}}$ for the three molecular weights of PI stars are 9.13, 13.88, and 18.27. If we also use the same value of $\tau_{\mathrm{e}}$ as for linear PI $\left(\tau_{\mathrm{e}}=6.0 \times 10^{-5} \mathrm{~s}\right)$, the predictions underestimate the longest relaxation times by approximately a factor of 2 (Fig 9a, b, solid lines). The best fit to experimental data are obtained using $\tau_{\mathrm{e}}=2 \times 10^{-4} \mathrm{~s}$ (Fig. 9a, b, broken line). Since the value of $\tau_{\mathrm{e}}$ for the linear chains was also obtained by fitting, it is not clear which of the two values of $\tau_{\mathrm{e}}$ should be considered the more accurate.

We have also compared the model with the experimental data for linear and star PI from Pearson et al. (1983). These linear polyisoprene data are for samples with three different molecular weights: $316 \mathrm{~K}, 513 \mathrm{~K}$, and $561 \mathrm{~K}$ with narrow molecular weight distributions at $75^{\circ} \mathrm{C}$ (corresponding to $\mathrm{N}_{\mathrm{en}}=60.77,98.65$, and 107.88). The data for star PI are reported for 8-arm

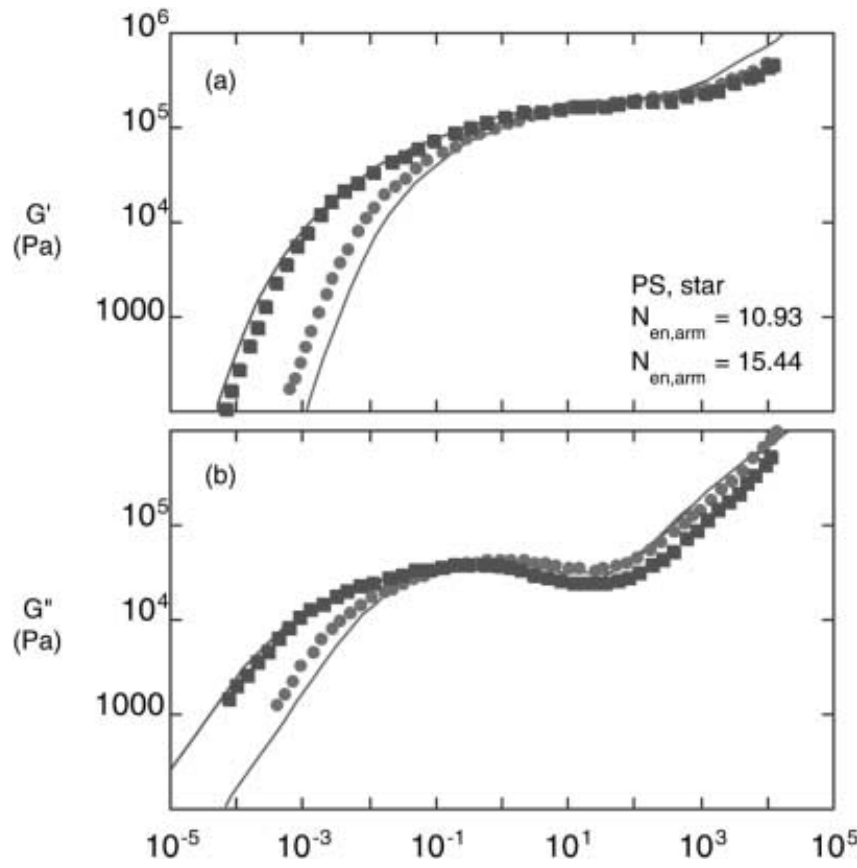

Fig. 7a, b Comparison of the model predictions of: a storage modulus $\left(\mathrm{G}^{\prime}\right)$; b loss modulus $\left(\mathrm{G}^{\prime \prime}\right)$ with experimental data for star polystyrenes at reference temperature $169.5^{\circ} \mathrm{C}$ (Graessley and Roover 1979). The molecular weights of the samples from right to left are $1090 \mathrm{~K}$ (6 arms) and $1027 \mathrm{~K}$ (4 arms), respectively. The solid lines are the model predictions using the literature values of the parameters: $\mathrm{G}_{\mathrm{N}}^{0}=2 \times 10^{5} \mathrm{~Pa}$ and $\tau_{\mathrm{e}}=1.96 \times 10^{-3} \mathrm{~s}$

stars with a molecular weight of $790 \mathrm{~K}\left(\mathrm{~N}_{\mathrm{en}}=18.99\right)$ at the same temperature. The comparisons of the model predictions with experimental data for the linear and star PI are shown in Figs. 10 and 11, respectively. We use the same literature values of $G_{N}^{0}$ and $M_{e}$ as for the

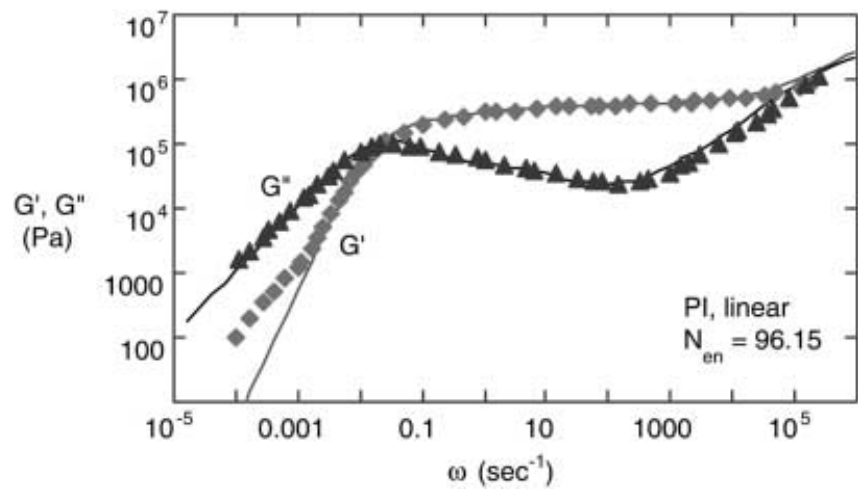

Fig. 8 Comparison of the model predictions of storage modulus $\left(\mathrm{G}^{\prime}\right)$ and loss modulus $\left(\mathrm{G}^{\prime \prime}\right)$ with experimental data for a linear polyisoprene with $\mathrm{MW}=500 \mathrm{~K}$ at $25^{\circ} \mathrm{C}$ (Fetters et al. 1993). The solid lines are the model predictions with the literature value of $\mathrm{G}_{\mathrm{N}}^{0}$ (i.e., $\mathrm{G}_{\mathrm{N}}^{0}=4.34 \times 10^{5} \mathrm{~Pa}$ ). Since the monomeric friction coefficient is not available in the literature, $\tau_{\mathrm{e}}$ is chosen to give the best fit to the experimental results. This gives $\tau_{\mathrm{e}}=6.0 \times 10^{-5} \mathrm{~s}$ 

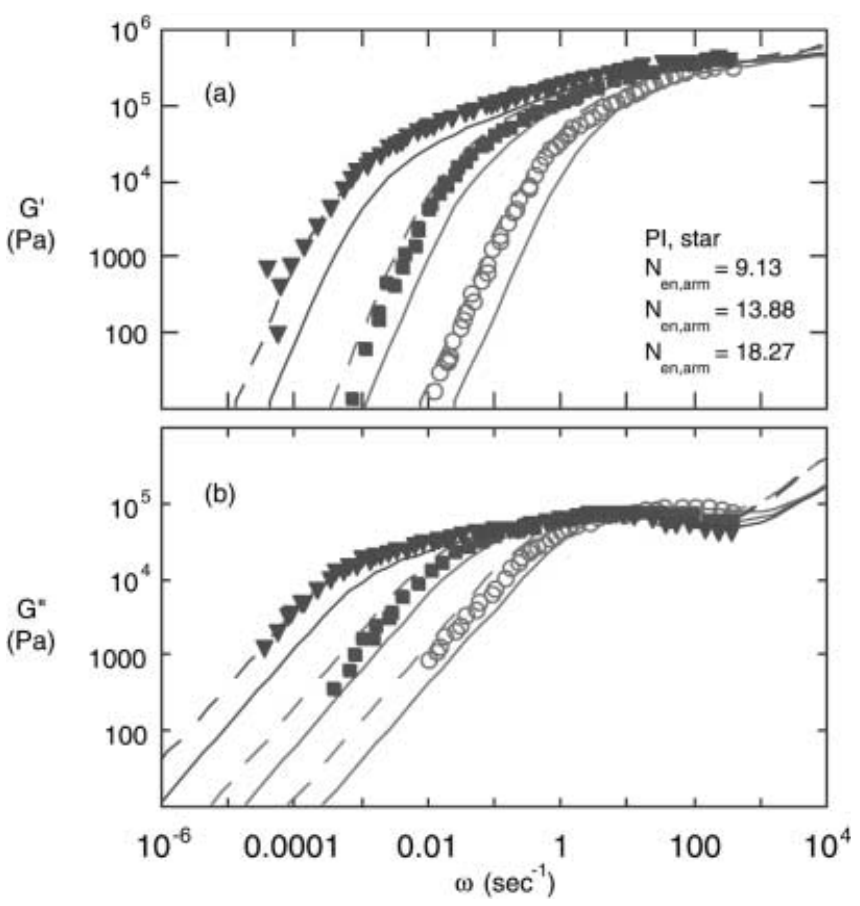

Fig. 9a, b Comparison of the model predictions of: a storage modulus, $\mathrm{G}^{\prime}$; b loss modulus, $\mathrm{G}^{\prime \prime}$, with experimental data for star polyisoprenes at $25{ }^{\circ} \mathrm{C}$ (Fetters et al. 1993). The arm molecular weights of the samples from right to left are $47.5 \mathrm{~K}, 72.2 \mathrm{~K}$, and $95 \mathrm{~K}$, respectively. The predictions from our model are represented by lines with the literature value of $\mathrm{G}_{\mathrm{N}}^{0}$ (i.e., $\mathrm{G}_{\mathrm{N}}^{0}=4.34 \times 10^{5} \mathrm{~Pa}$ ). The solid lines were obtained using the same $\tau_{\mathrm{e}}$ as for the linear PI, namely $\tau_{\mathrm{e}}=6.0 \times 10^{-5} \mathrm{~s}$. However, $\tau_{\mathrm{e}}$ that gives the best fit to the experimental data is $\tau_{\mathrm{e}}=2.0 \times 10^{-4} \mathrm{~s}$ (broken lines)

comparison with Fetters' data mentioned above for both linear and star PI. Since $\zeta$ is unavailable at $75{ }^{\circ} \mathrm{C}$ as it was at $25^{\circ} \mathrm{C}$, the value of $\tau_{\mathrm{e}}=4 \times 10^{-5} \mathrm{~s}$, which gives the best fit to the linear PI data, was chosen. Surprisingly, for the star PI, the same value of $\tau_{\mathrm{e}}$ as for the linears gives a large discrepancy in the prediction of the star data. In order to obtain the best fit, $\tau_{\mathrm{e}}$ was adjusted to a value of $5 \times 10^{-6} \mathrm{~s}$ (broken lines) which is approximately eight times lower than the value of $\tau_{\mathrm{e}}$ used for linear PI at the same temperature. This large discrepancy is particularly surprising in view of the agreement in the value of $\tau_{\mathrm{e}}$ required to fit both star and linear data for the three other sets of samples, including polybutadiene, polystyrene, and even another set of polyisoprene samples at a different temperature. At present, we have no explanation for the discrepancy for this polyisoprene at $75^{\circ} \mathrm{C}$.

\section{Bidisperse polymers - case studies}

Since the model can capture the linear viscoelastic properties of monodisperse polymers in most cases with

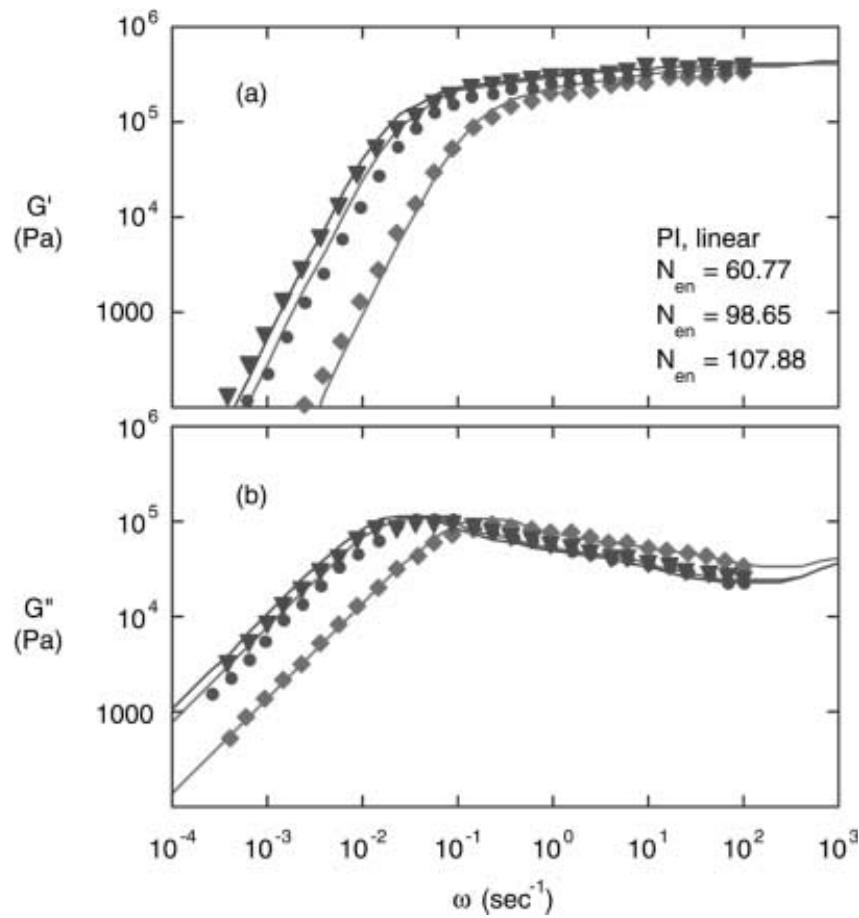

Fig. 10a, b Comparison of the model predictions of: a storage modulus, $G^{\prime}$; b loss modulus, $G^{\prime \prime}$, with experimental data for linear polyisoprenes at $75{ }^{\circ} \mathrm{C}$ (Pearson et al. 1983). The molecular weights of the samples from right to left are $316 \mathrm{~K}, 513 \mathrm{~K}$, and $561 \mathrm{~K}$, respectively. The solid lines are the model predictions with the literature value of $\mathrm{G}_{\mathrm{N}}^{0}$ (i.e., $\mathrm{G}_{\mathrm{N}}^{0}=4.34 \times 10^{5} \mathrm{~Pa}$ ). Since the monomeric friction coefficient is not available in literature, $\tau_{\mathrm{e}}$ is chosen to give the best fit to the experimental results. This gives $\tau_{\mathrm{e}}=4 \times 10^{-5} \mathrm{~s}$

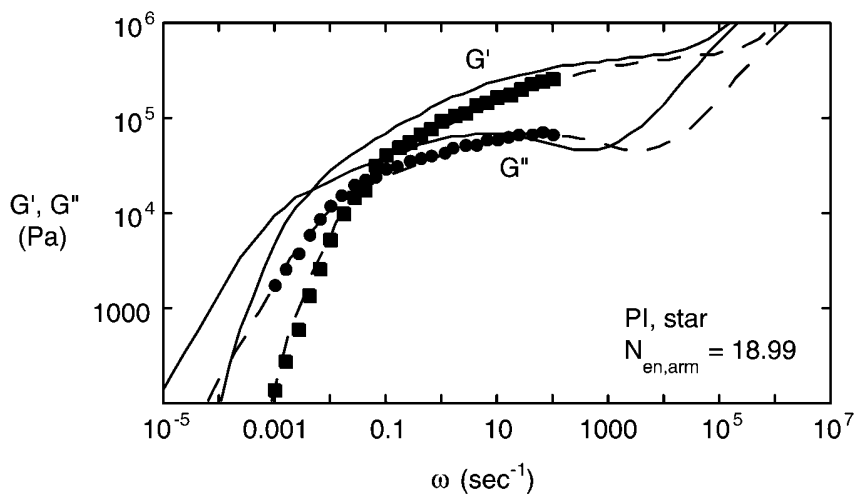

Fig. 11 Comparison of the model predictions of storage modulus $\left(G^{\prime}\right)$ and loss modulus $\left(G^{\prime \prime}\right)$ with experimental data for 8-arm star polyisoprenes $\mathrm{MW}=790 \mathrm{~K}$ at $75^{\circ} \mathrm{C}$ (Pearson et al. 1983). The predictions from our model are represented by lines with the literature value of $\mathrm{G}_{\mathrm{N}}^{0}$ (i.e., $\mathrm{G}_{\mathrm{N}}^{0}=4.34 \times 10^{5} \mathrm{~Pa}$ ). The solid lines were obtained using the same $\tau_{\mathrm{e}}$ as for the linear PI, namely $\tau_{\mathrm{e}}=4 \times 10^{-5} \mathrm{~s}$. However, $\tau_{\mathrm{e}}$ that gives the best fit to the experimental data is $\tau_{\mathrm{e}}=$ $5 \times 10^{-6}$ s (broken lines)

no adjustable parameters, we now move on to test the model for binary blends of low and high molecular weight entangled polymers of the same architecture. For 
linear-linear blends, data are available for polybutadiene and polystyrene. For star-star blends, the comparison is only possible for a single set of polyisoprenes. The results show that the model can capture the mixing behavior of those polymers very well, as described in detail below.

\section{Linear-linear binary blends}

Polybutadiene. There are quite a few experimental data sets to test for polybutadiene bidisperse blends. One is from Rubinstein and Colby $(1988)$ at $30{ }^{\circ} \mathrm{C}$. It consists of the mixture of $\mathrm{MW}=70.9 \mathrm{~K}\left(\mathrm{~N}_{\mathrm{en}}=31.25\right)$ and $335 \mathrm{~K}$ $\left(\mathrm{N}_{\mathrm{en}}=156.46\right)$ with volume fractions of the high molecular weight $\left(\mathrm{x}_{1}\right)$ of $0.0,0.638,0.768,0.882$, and 1.0 . The model parameters, $\mathrm{G}_{\mathrm{N}}^{0}$ and $\tau_{\mathrm{e}}$ at $25^{\circ} \mathrm{C}$, are taken to be the same as for monodisperse PBD, already discussed. Since the test temperature is $30^{\circ} \mathrm{C}$, the WLF function is applied to obtain a value of $\tau_{\mathrm{e}}=9.315 \times 10^{-7} \mathrm{~s}$ at $30^{\circ} \mathrm{C}$. As shown in Fig. 12, the model predicts $G^{\prime \prime}$ for this experimental data set very well. Another experimental data set was taken from Struglinski et al. (1985) which reports extensive rheological properties for three sets of

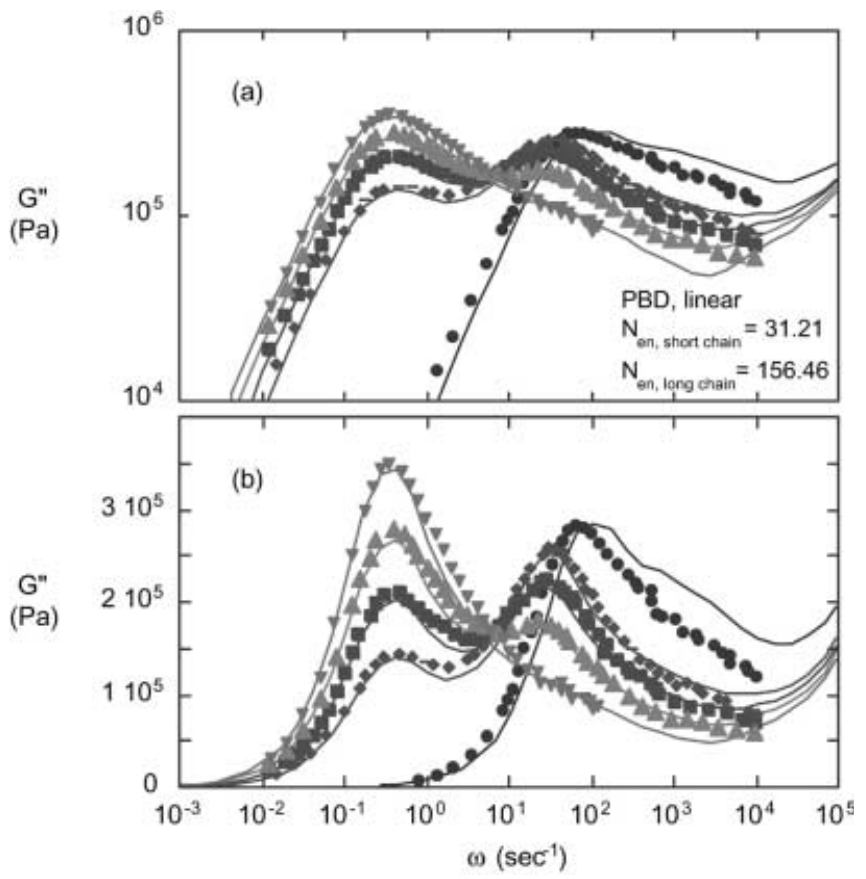

Fig. 12a, b Comparison of the model predictions of loss modulus, $\mathrm{G}^{\prime \prime}$, with experimental data for linear-linear blends of polybutadienes $\left(\mathrm{MW}=70.9 \mathrm{~K} / 335 \mathrm{~K}\right.$ ) at $30^{\circ} \mathrm{C}$ (Rubinstein and Colby 1988). The comparisons are made on: a log-log; $\mathbf{b} \log$-linear scales. The volume fractions of the high molecular weight component $\left(\mathrm{x}_{1}\right)$ from right to left are $0.0,0.638,0.768,0.882$, and 1.0 , respectively. The solid lines are the model predictions using the literature parameters $\mathrm{G}_{\mathrm{N}}^{0}=1.25 \times$ $10^{6} \mathrm{~Pa}$ and $\tau_{\mathrm{e}}=9.315 \times 10^{-7} \mathrm{~s}$
PBD mixtures at $25^{\circ} \mathrm{C}$. The first set is $41 \mathrm{~L} / 174 \mathrm{~L}$, which is a set of mixtures of PBD with $\mathrm{MW}=39 \mathrm{~K}$ $\left(\mathrm{N}_{\mathrm{en}}=17.19\right)$ and $181 \mathrm{~K}\left(\mathrm{~N}_{\mathrm{en}}=79.77\right)$, with $\mathrm{x}_{1}=0.0$, $0.1,0.3,0.5,0.7$, and 1.0. The second set, $98 \mathrm{~L} / 435 \mathrm{~L}$, consists of blends of higher molecular weights, $\mathrm{MW}=92.5 \mathrm{~K}\left(\mathrm{~N}_{\mathrm{en}}=40.77\right)$ and $450 \mathrm{~K}\left(\mathrm{~N}_{\mathrm{en}}=198.33\right)$, with $\mathrm{x}_{1}=0.0,0.3,0.48,0.8$, and 1.0 . The third set $(41 \mathrm{~L} /$ 435L) contains mixtures of the lowest molecular weight, $\mathrm{MW}=39 \mathrm{~K} \quad\left(\mathrm{~N}_{\mathrm{en}}=17.19\right)$, with the highest one, $\mathrm{MW}=450 \mathrm{~K}\left(\mathrm{~N}_{\mathrm{en}}=198.33\right)$, with the following volume fractions of the high molecular weight: $\mathrm{x}_{1}=0.0,0.26$, $0.56,0.8$, and 1.0. The model parameters are again the same as the monodisperse PBD at $25^{\circ} \mathrm{C}$ (i.e., $\tau_{\mathrm{e}}=1.51 \times 10^{-6} \mathrm{~s}$ and $\mathrm{G}_{\mathrm{N}}^{0}=1.25 \times 10^{6} \mathrm{~Pa}$ ). The comparison for the first, second, and third sets are shown in Figs. 13, 14, and 15, respectively.

As can be seen in Fig. 13 for $41 \mathrm{~L} / 174 \mathrm{~L}$, the model can predict $G^{\prime}$ and $G^{\prime \prime}$ very well for all concentrations of the high molecular weight component $(174 \mathrm{~L})$. For the second set $(98 \mathrm{~L} / 435 \mathrm{~L})$ in Fig. 14 and the third set $(41 \mathrm{~L} /$ 174L) in Fig. 15, the model cannot predict the behavior for the very high molecular weight component $435 \mathrm{~L}$ $\left(\mathrm{N}_{\mathrm{en}}=198.33\right)$ well using the literature value of $\tau_{\mathrm{e}}$. In order to get the best fit for the high molecular weight

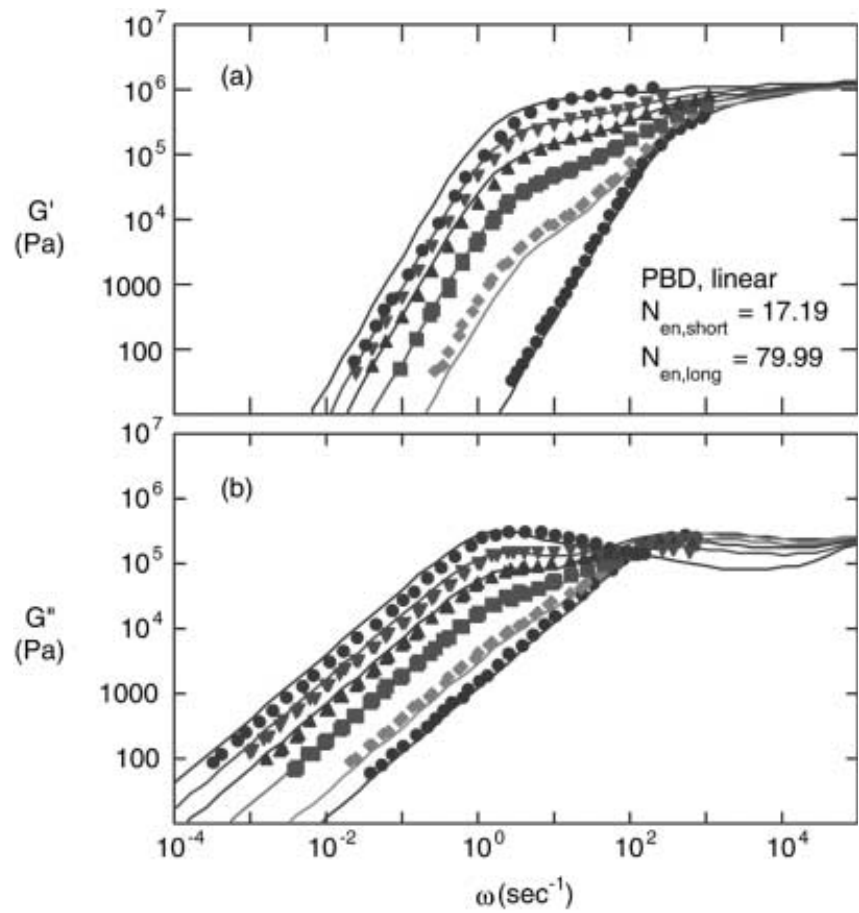

Fig. 13a, b Comparison of the model predictions of: a storage modulus, $\mathrm{G}^{\prime}$; b loss modulus, $\mathrm{G}^{\prime \prime}$, with experimental data for linearlinear blend of polybutadienes $(41 \mathrm{~L} / 174 \mathrm{~L})$ at $25^{\circ} \mathrm{C}$. (Struglinski et al. 1985) The volume fractions of the high molecular weight component $\left(\mathrm{x}_{1}\right)$ from right to left are $0.0,0.1,0.3,0.5,0.7$, and 1.0 , respectively. The solid lines show the corresponding model predictions using the literature parameters $\mathrm{G}_{\mathrm{N}}^{0}=1.25 \times 10^{6} \mathrm{~Pa}$ and $\tau_{\mathrm{e}}=1.51 \times 10^{-6} \mathrm{~s}$ 


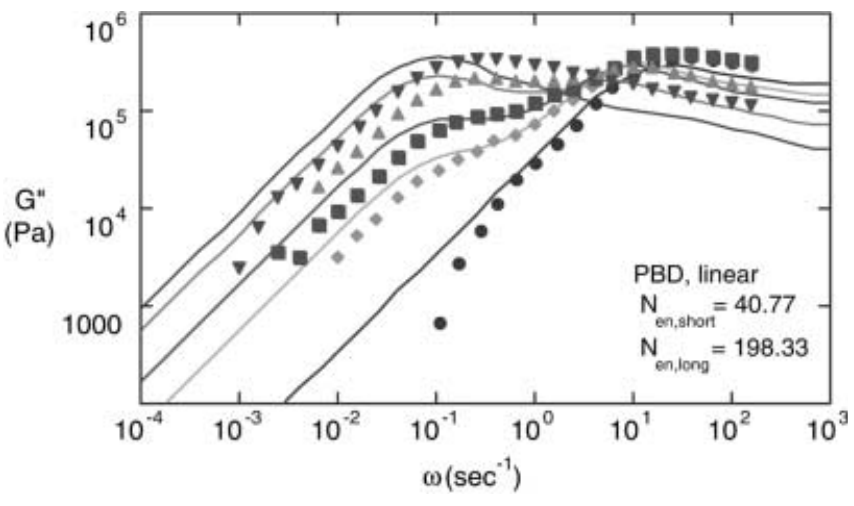

Fig. 14 Comparison of the model predictions of loss modulus, $G^{\prime \prime}$, with experimental data for linear-linear blends of polybutadienes (98L/435L) at $25^{\circ} \mathrm{C}$ (Struglinski et al. 1985). The volume fractions of the high molecular weight component $\left(\mathrm{x}_{1}\right)$ from right to left are $0.0,0.3$, $0.48,0.8$, and 1.0, respectively. The solid lines show the corresponding model predictions using the literature parameters $\mathrm{G}_{\mathrm{N}}^{0}=1.25 \times 10^{6} \mathrm{~Pa}$ and $\tau_{\mathrm{e}}=1.51 \times 10^{-6} \mathrm{~s}$

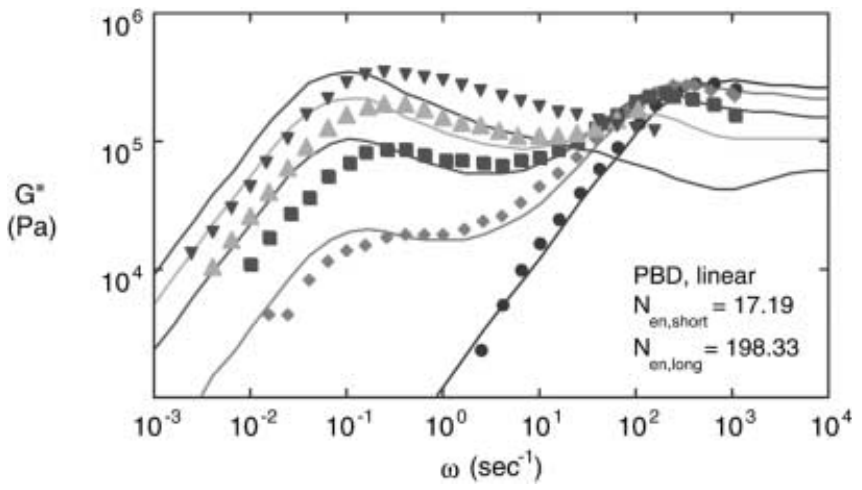

Fig. 15 Comparison of the model predictions of loss modulus, $\mathrm{G}^{\prime \prime}$, with experimental data for linear-linear blends of polybutadienes $(41 \mathrm{~L} / 435 \mathrm{~L})$ at $25^{\circ} \mathrm{C}$ (Struglinski et al. 1985). The volume fractions of the high molecular weight component $\left(\mathrm{x}_{1}\right)$ from right to left are 0.0 , $0.26,0.56,0.8$, and 1.0, respectively. The solid lines show the corresponding model predictions using the literature parameters $\mathrm{G}_{\mathrm{N}}^{0}=$ $1.25 \times 10^{6} \mathrm{~Pa}$ and $\tau_{\mathrm{e}}=1.51 \times 10^{-6} \mathrm{~s}$

component, $\tau_{\mathrm{e}}$ would need to be shifted to $0.9 \times 10^{-6} \mathrm{~s}$ which is about 1.7 time smaller than the literature value at this temperature. We suspect that the model starts to overestimate the longest relaxation time at very high molecular weight. This might also explain the discrepancy of $\tau_{\mathrm{e}}$ for the monodisperse linear and star PI in Figs. 8 and 9 in that $\mathrm{N}_{\text {en }}$ for linear PI in Fig. 8 $\left(\mathrm{N}_{\mathrm{en}}=96.15\right)$ may be too high, thus giving a higher value of the best-fit $\tau_{\mathrm{e}}$. Apart from this, the rheological predictions of the mixtures using the literature value of $\tau_{\mathrm{e}}$, i.e., $1.51 \times 10^{-6} \mathrm{~s}$, agree well with the data as shown in Fig. $14 \mathrm{a}, \mathrm{b}$ for $98 \mathrm{~L} / 435 \mathrm{~L}$ and Fig. $15 \mathrm{a}, \mathrm{b}$ for $41 \mathrm{~L} /$ $435 \mathrm{~L}$, respectively.

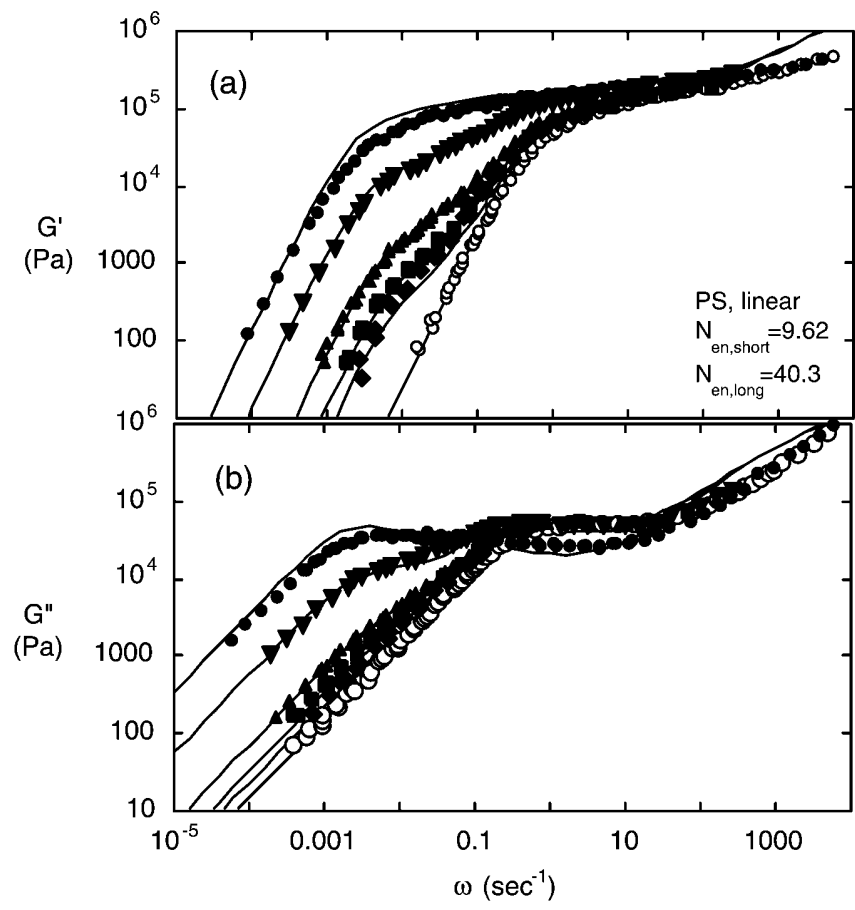

Fig. 16a, b Comparison of the model predictions of: a storage modulus, $\mathrm{G}^{\prime}$; b loss modulus, $\mathrm{G}^{\prime \prime}$, with experimental data for linearlinear blend of polystyrenes $(\mathrm{MW}=160 \mathrm{~K} / 670 \mathrm{~K})$ at $160{ }^{\circ} \mathrm{C}$. (Montfort et al. 1979) The volume fractions of the high molecular weight component $\left(\mathrm{x}_{1}\right)$ from right to left are $0.0,0.05,0.1,0.2,0.5$, and 1.0 , respectively. The solid lines show the corresponding model predictions using the literature value of $\mathrm{G}_{\mathrm{N}}^{0}$ (i.e., $\mathrm{G}_{\mathrm{N}}^{0}=0.2 \times 10^{6} \mathrm{~Pa}$ ). $\tau_{\mathrm{e}}=0.01 \mathrm{~s}$ was adjusted to give the best fit to the experimental result

Polystyrene. The experimental binary blends of polystyrene at $160{ }^{\circ} \mathrm{C}$ are taken from Montfort et al. (1979), who reported the rheological properties for a set of polystyrene mixtures of $\mathrm{MW}=160 \mathrm{~K}\left(\mathrm{~N}_{\mathrm{en}}=9.62\right)$ and $670 \mathrm{~K}\left(\mathrm{~N}_{\mathrm{en}}=40.3\right)$ with $\mathrm{x}_{1}=0.0,0.05,0.1,0.2,0.5$, and 1.0. For the model parameters, we use the same $G_{N}^{0}, M_{e}$, and $a$ as for monodisperse PS mentioned earlier. For $\zeta$, since the temperature of $160{ }^{\circ} \mathrm{C}$ is close to the glass transition temperature $\left(\mathrm{T}_{\mathrm{g}}=100{ }^{\circ} \mathrm{C}\right)$ and $\zeta$ becomes too temperature-sensitive for the correlation of Majeste et al. used earlier to be accurate, we left $\tau_{\mathrm{e}}$ to be the only fitting parameter for this set. As can be seen from Fig. 16a, b, the predictions capture the binary behavior of this set of mixtures reasonably well at every concentration using the fitted value $\tau_{\mathrm{e}}=0.01 \mathrm{~s}$.

\section{Star-star blends}

The single set of experimental data for star-star blends found in the literature is due to Blottière et al. (1998). The data are for 3-arm PI star blends of two different molecular weights, $\mathrm{MW} / \mathrm{arm}=28 \mathrm{~K}\left(\mathrm{~N}_{\mathrm{en}}=5.385\right)$ and $\mathrm{MW} / \mathrm{arm}=144 \mathrm{~K}\left(\mathrm{~N}_{\mathrm{en}}=27.69\right)$, with $\mathrm{x}_{1}=0.0,0.2,0.5$, 


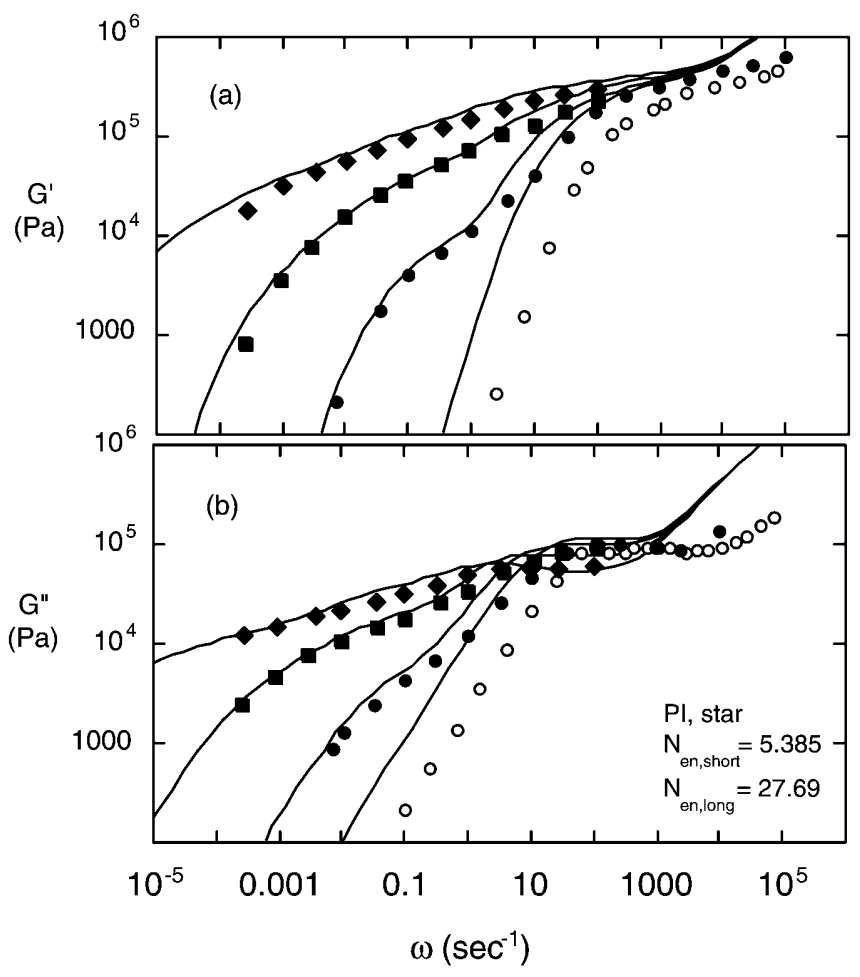

Fig. 17a, b Comparison of the model predictions of: a storage modulus, $G^{\prime}$; b loss modulus, $G^{\prime \prime}$, with experimental data for star-star blend of polyisoprene $\left(\mathrm{MW}_{\mathrm{arm}}=28 \mathrm{~K} / 144 \mathrm{~K}\right)$ at $25^{\circ} \mathrm{C}$ (Blottiére et al. 1998). The volume fraction of the high molecular weight component $\left(\mathrm{x}_{1}\right)$ from right to left are $0.0,0.2,0.5$, and 0.8 , respectively. The solid lines from left to right show the corresponding model predictions using the same model parameters as for the broken line in Fig. 9 , i.e., $\mathrm{G}_{\mathrm{N}}^{0}=1.25 \times 10^{6} \mathrm{~Pa}$ and $\tau_{\mathrm{e}}=2 \times 10^{-4} \mathrm{~s}$

0.8 at $25{ }^{\circ} \mathrm{C} .\left(\mathrm{x}_{1}=1.0\right.$ is omitted because no accurate experimental data are reported for pure PI with $\mathrm{MW} /$ arm $=144 \mathrm{~K}$.) Since the temperature is the same as for monodisperse star PI mentioned earlier, we use the same model parameters as reported there (i.e., $\mathrm{G}_{\mathrm{N}}^{0}=4.34 \times$ $10^{5} \mathrm{~Pa}$ and $\tau_{\mathrm{e}}=2 \times 10^{-4} \mathrm{~s}$ ). As shown in Fig. $17 \mathrm{a}, \mathrm{b}$, the model can predict the binary behavior of the star blends well without adjustable parameters. However, the prediction for the short chain $(\mathrm{MW} / \mathrm{arm}=28 \mathrm{~K})$ is off by a factor of 4 , which is expected because of its small number of entanglements.

\section{Polydisperse linear polymers - case studies}

Experimental data sets for polydisperse polymers which provide a good test of the model are those from Wasserman and Graessley (1992). They prepared two polystyrene samples, M1 $\quad($ P.I. $=2.3)$ and M2 $($ P.I. $=2.57)$. These consist of 11 and 13 molecular weight components, respectively. M2 has the same components as M1 except that M2 has small additional amounts of high molecular weight components. The compositions of the mixtures are shown in Table 3 .

The comparisons between the experimental data and the model predictions for $\mathrm{M} 1$ and $\mathrm{M} 2$ are shown in Fig. 18. The model parameter $G_{\mathrm{N}}^{0}$ is again taken from the literature $\left(\mathrm{G}_{\mathrm{N}}^{0}=0.2 \mathrm{MPa}\right)$. For $\zeta$, since the temperature $\left(150^{\circ} \mathrm{C}\right)$ is again too low for the Majeste's correlation used earlier to be accurate, we left $\tau_{\mathrm{e}}$ to be the only fitting parameter for this set, and obtained $\tau_{\mathrm{e}}=5 \times 10^{-2} \mathrm{~s}$. As can be seen, the model can predict well the effect of the small fractions of the high molecular weight components in M2.

A lesser degree of polydispersity was also accounted for accurately earlier for the linear PBD with $\mathrm{MW}=201 \mathrm{~K}(\mathrm{P} . \mathrm{I} .=1.27)$ by breaking the distribution of the PBD into 20 discrete components (the first curve

Table 3 Composition of polystyrene mixtures M1 and M2

\begin{tabular}{rll}
\hline M & \multicolumn{2}{l}{ Weight Fractions } \\
\cline { 2 - 3 } & M1 & M2 \\
\hline 2,980 & 0.001 & 0.001 \\
5,570 & 0.002 & 0.002 \\
9,100 & 0.004 & 0.004 \\
19,600 & 0.008 & 0.008 \\
37,900 & 0.030 & 0.030 \\
96,400 & 0.150 & 0.148 \\
190,000 & 0.260 & 0.257 \\
355,000 & 0.358 & 0.353 \\
706,000 & 0.140 & 0.139 \\
$1,090,000$ & 0.039 & 0.038 \\
$2,890,000$ & 0.008 & 0.010 \\
$3,840,000$ & & 0.007 \\
$4,480,000$ & & 0.003 \\
\hline
\end{tabular}

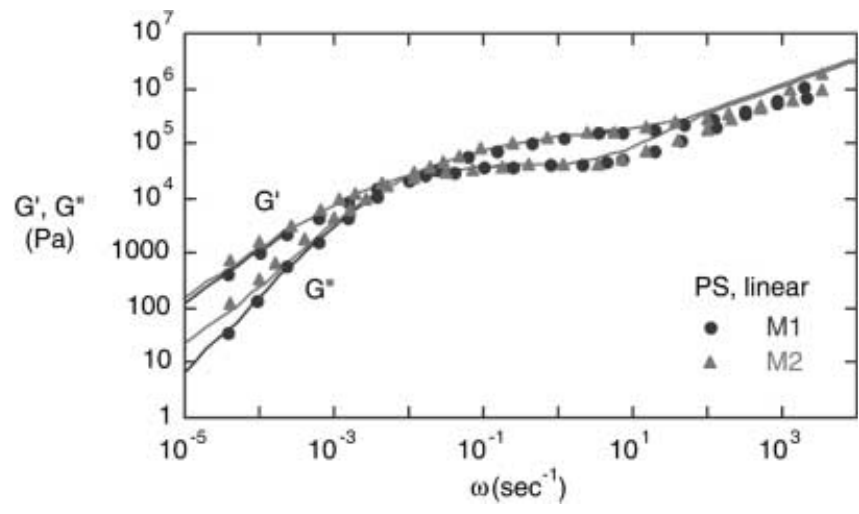

Fig. 18 Comparison of the model predictions of storage modulus $\left(\mathrm{G}^{\prime}\right)$ and loss modulus $\left(\mathrm{G}^{\prime \prime}\right)$ with experimental data for linear polydisperse polystyrenes (M1 and M2) at the reference temperature $150{ }^{\circ} \mathrm{C}$ (Wasserman and Graessley et al. 1992). The solid lines are the model predictions with the parameter $\mathrm{G}_{\mathrm{N}}^{0}=2 \times 10^{5} \mathrm{~Pa}$ (Fetters et al. 1994). $\tau_{\mathrm{e}}=5.0 \times 10^{-2} \mathrm{~s}$ was adjusted to give the best fit to the experimental results 


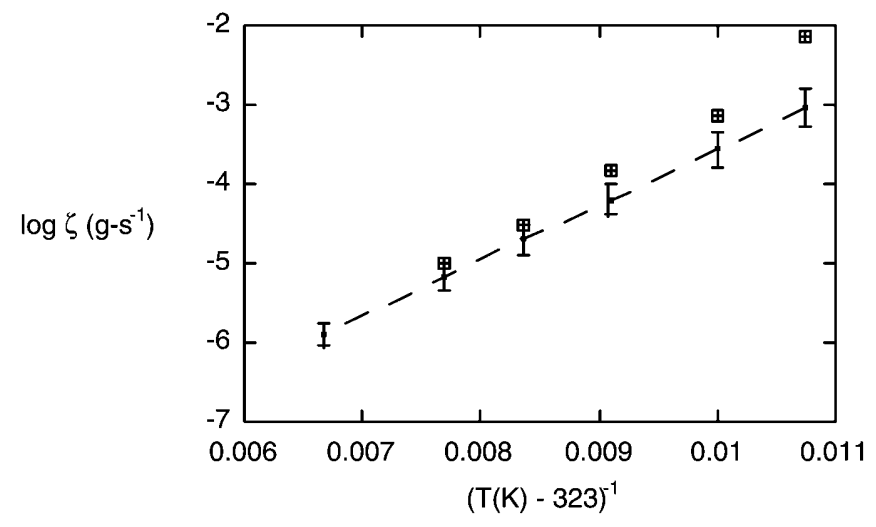

Fig. 19 Comparison of the monomeric friction coefficient, $\zeta$, for polystyrenes from Majeste's correlation (broken line with error bars) and from the best fit of the model to the rheological data (symbol)

on the left in Fig. 3a, b). As mentioned earlier, the model gave a good prediction of $\mathrm{G}^{\prime}$ and $\mathrm{G}^{\prime \prime}$ for this somewhat polydisperse polymer.

In this paper, the rheological predictions of PS were considered at various temperatures, i.e., $180{ }^{\circ} \mathrm{C}$, $169.5^{\circ} \mathrm{C}, 160{ }^{\circ} \mathrm{C}$, and $150{ }^{\circ} \mathrm{C}$. Although the correlation of $\zeta$ as a function of temperature for PS is available from Majeste et al., the correlated values only give good predictions to experimental data at $180{ }^{\circ} \mathrm{C}$ and $169.5^{\circ} \mathrm{C}$. At lower temperatures, the $\zeta$ s that give the best fits to PS data start to deviate from Majeste's values. By converting the best-fit $\tau_{\mathrm{e}}$ for PS at $160{ }^{\circ} \mathrm{C}$ and $150{ }^{\circ} \mathrm{C}$ back to $\zeta$ using the correlation $\zeta a^{2} \mathrm{M}_{\mathrm{e}} / 3 \pi^{2} \mathrm{k}_{\mathrm{B}} \mathrm{TM}_{0}$ mentioned earlier, we can compare Majeste et al.'s predicted values of $\zeta$ with the values of $\zeta$ that give the best fits to experimental data. As shown in Fig. 19, the best-fit $\zeta$ (symbols) starts to deviate from the values of Majeste et al. (broken line with error bars) at low temperature. We suspect that, for temperatures close to $\mathrm{T}_{\mathrm{g}}\left(100^{\circ} \mathrm{C}\right), \zeta$ becomes too temperature-sensitive for the correlation of Majeste et al. to be accurate.

\section{Conclusion}

We have systematically compared the model predictions of the "dual constraint" model, a general theory for the linear viscoelastic properties of entangled polymers with literature data for several monodisperse polymer melts, including polybutadiene, polyisoprene, and polystyrene, for both linear and star architectures. The predictions are also extended to the rheological behavior of bidisperse and polydisperse polymers. The theory is that of Mead, Van Dyke et al. (2000) extended by including early-time contour-length fluctuations and constraint-release Rouse processes (Viovy et al. 1991; Milner et al. 1998), as well as Rouse modes modified using the "fragmented spectrum" of Milner and McLeish (1998). The model parameters $\tau_{\mathrm{e}}$ (calculated from $\zeta$ and $a$ ) and $\mathrm{G}_{\mathrm{N}}^{0}$, which are independent of polymer molecular weight and architecture, are obtained from the literature and are taken to be identical for polymers with the same chemical composition and at the same temperature, regardless of their architectures. The model appears to predict the monodisperse, bidisperse, and polydisperse literature data well without adjustable parameters, except for the case of polystyrene at low temperature and polyisoprene, for which reliable $\zeta$ was not available in the literature which necessitated obtaining the value by fitting. Nevertheless, by comparing the model with experimental data, we observed a few weaknesses of the predictions in both star and linear polymers. For star polymers, we find that the model fails at low $\mathrm{N}_{\mathrm{en}}$ and suspect that the number of arms might affect the terminal relaxation time (which is not predicted by the model). In some cases, especially in polyisoprene, the star polymers seem to need a higher equilibrium time $\tau_{\mathrm{e}}$ than the linear counterpart. For linear polymers, for a best fit to the data, the model seems to need a different value of $\tau_{\mathrm{e}}$ when the number of entanglements is either too low or too high. The problem may arise either from some inaccuracy of the "tube" concept at low numbers of entanglements or from the form of the prefactor $\left(\tau_{\mathrm{R}}\right)$ used in the expression for the contourlength fluctuation in Eq. (2). (We note that Pearson and Helfand 1984 used a weaker departure of the prefactor on $\mathrm{N}_{\mathrm{en}}$, namely $\mathrm{N}_{\mathrm{en}}{ }^{3 / 2}$, and we have found that this form of prefactor can improve the predictions at the low and the high ends of the molecular-weight range.) For binary blends, the "dual constraint" model works almost perfectly for both linear-linear blends and star-star blends at all concentrations. The outstanding point of the model is that it can be universally applied to the linear and star polymers, whether monodisperse, bidisperse, or polydisperse, without introducing additional parameters other than the ones required for the original Doi-Edwards model. Moreover, the conceptual framework underlying the model can be further extended to the regime of nonlinear viscoelasticity. In addition, if experimental linear viscoelastic data for well-entangled polymers are provided at several different temperatures, the model predictions can be used as a means of obtaining a reliable correlation of the monomeric friction coefficient, $\zeta$, with temperature.

Acknowledgements RGL is grateful for support for this work from the National Science Foundation, grant \# DMR 9807262. CP is grateful for a Royal Thai Fellowship from the government of Thailand. 


\section{References}

Adams CH, Hutchings LR, Klein PG, McLeish TCB, Richards RW (1996) Synthesis and dynamic rheological behavior of polybutadiene. Macromolecules 29:5717-5722

Ball RC, McLeish TCB (1989) Dynamic dilution and the viscosity of star polymer melts. Macromolecules 22:1911-1913

Baumgaertel M, De Rosa ME, Machado J, Masse M, Winter HH (1992) The relaxation time spectrum of nearly monodisperse polybutadiene melts. Rheol Acta 31:75-82

Blottière B, McLeish TCB, Hakiki RN, Young RN, Milner ST (1998) The rheology of bimodal blends of star polymer melts. Macromolecules 31:9295-9304

des Cloizeaux J (1988) Double reptation vs. simple reptation in polymer melts. $\mathbf{J}$ Europhys Lett 5:437-442

de Gennes PG (1971) Reptation of a polymer chain in the presence of fixed obstacles. J Chem Phys 55:572-579

Doi M, Edwards SF (1986) The theory of polymer dynamics. Clarendon, Oxford

Doi M, Kuzuu NY (1980) Rheology of Star Polymers in Concentrated Solutions and Melts. J Polym Sci, Polym Lett Ed 18:775-780

Doi M, Edwards SF (1978) Dynamics of concentrated polymer systems, pts $1-3$. J Chem Soc, Faraday Trans II 74:1789-1832

Doi M, Edwards SF (1979) Dynamics of concentrated polymer systems, pt 4 . Rheological properties. J Chem Soc, Faraday Trans II. 75:38-54

Ferry JD (1980) Viscoelastic properties of polymers. Wiley, New York

Fetters LJ, Kiss AD, Pearson DS, Quack GF, Vitus FJ (1993) Rheological behavior of star-shaped polymers. Macromolecules 26:647-654

Fetters LJ, Lohse DJ, Richter D, Witten TA, Zirkel A (1994) Connection between polymer molecular weight, den- sity, chain dimensions, and melt viscoelastic properties. Macromolecules 27:4639-4647

Graessley WW, Roovers J (1979) Melt rheology of four-arm and six-arm star polystyrenes. Macromolecules 12:959965

Klein J (1986) Dynamics of entangled linear, branched, and cyclic polymers. Macromolecules 19:105-118

Majeste JC, Montfort JP, Allal A, Marin G (1998) Viscoelasticity of low molecular weight polymers and the transition to the entangled regime. Rheol Acta 37:486-499

Mead DW, Van Dyke TJ, Larson RG, Doi M (2000) Unified theory of relaxation in star and linear polymers (in preparation)

Milner ST (1996) Relating the shear-thinning curve to the molecular weight distribution in linear polymer melts. Macromolecules 40:303-315

Milner ST, McLeish TCB (1997) Parameter-free theory for stress relaxation in star polymer melts. Macromolecules 30:2159-2166

Milner ST, McLeish TCB (1998) Reptation and contour-length fluctuations in melts of linear polymers. Phys Rev Lett 81:725-728

Milner ST, McLeish TCB, Young RN, Hakiki A, Johnson JM (1998) Dynamic dilution, constraint-release, and starlinear blends. Macromolecules 31:9345-9353

Montfort JP, Marin G, Arman J, Monge PH (1979) Viscoelastic properties of high molecular weight polymers in the molten state II. Influence of the molecular weight distribution on linear viscoelastic properties. Rheol Acta 18:623-628

Montfort JP, Marin G, Monge PH (1984) Effects of constraint release on the dynamics of entangled linear polymer melts. Macromolecules 17:1551-1560
Pearson DS, Helfand E (1984) Viscoelastic properties of star-shaped polymers. Macromolecules 17:888-895

Pearson DS, Mueller SJ, Fetters LJ, Hadjichristidis N (1983) Comparison of the rheological properties of linear and star-branched polyisoprenes in shear and elongational flows. J Polym Sci, Polym Phys Ed 21:2287-2298

Rubinstein M, Colby RH (1988) Self-consistent theory of polydisperse entangled polymers: linear viscoelasticity of binary blends. J Chem Phys 89(8):52915306

Schausberger A, Schindlauer G, JaneschitzKriegl H (1985) Linear elastico-viscous properties of molten standard polystyrenes. I. Presentation of complex moduli; role of short range structural parameters. Rheol Acta 24:220-227

Struglinski MJ, Graessley GG, Fetters L (1985) Effects of polydispersity on the linear viscoelastic properties of entangled polymers. 1. Experimental observations for binary mixtures of linear polybutadiene. Macromolecules 18:2630-2643

Tsenoglou C (1987) Viscoelasticity of binary homopolymer blends. ACS Polym Prepr 28:185-186

Viovy JL, Rubinstein M, Colby RH (1991) Constraint release in polymer melts: tube reorganization versus tube dilution. Macromolecules 24:3587-3596

Wasserman SH, Graessley WW (1992) Effects of polydispersity on linear viscoelasticity in entangled polymer melts. J Rheol 36:543-572

Watanabe H, Takashi S, Kotaka T (1985) Entanglements in linear polystyrenes. Macromolecule 18:1436-1442

Watanabe H, Yamazaki M, Yoshida $\mathrm{H}$, Kotaka T (1991) Viscoelastic properties of binary blends of linear polymers: further examination of constraint release models. Macromolecule 24:55735581 